\title{
Biocatalytic Synthesis of Lipophilic Baicalin Derivatives as
}

\section{Antimicrobial Agents}

\author{
Xuan Xin ${ }^{\dagger}$, Mengmeng Zhang ${ }^{\dagger}$, Xiao-Feng $\mathbf{L i}^{* \dagger}$, Guanglei Zhao* ${ }^{*}$
}

\section{Affiliation:}

$\dagger$ College of Food Science and Engineering, South China University of Technology, Wushan Road 381, Guangzhou, Guangdong, 510640, China

$\S$ State Key Laboratory of Pulp and Paper Engineering, South China University of Technology, Wushan Road 381, Guangzhou, Guangdong, 510640, China

Corresponding authors:

*Dr. Xiaofeng Li, School of Food Sciences and Engineering, South China University of Technology, Wushan Road 381, Guangzhou, Guangdong, China Tel: (+86)20-22236819; E-mail: xflibio@scut.edu.cn ; Fax: (+86)20-87112853

*Dr. Guanglei Zhao, State Key Laboratory of Pulp and Paper Engineering, South China University of Technology, Wushan Road 381, Guangzhou, Guangdong, China Tel: (+86)20-87111770; E-mail: glzhao@scut.edu.cn; Fax: (+86)20-87111770

\section{Content:}

Structural Information

Table S1-S2

Figure S1-S39

HPLC chromatography, ${ }^{1} \mathrm{H}$ NMR and ${ }^{13} \mathrm{C}$ NMR spectra of compounds 1 , 2a-2k
PS7-S8

PS9-S35
PS2-S6

(Figure S4-S39) 


\section{Structural Information}

Baicalin (1). ${ }^{1} \mathrm{H}$ NMR (400 MHz, DMSO- $\left.d 6\right) \delta 12.60(\mathrm{~s}, 1 \mathrm{H}), 8.68(\mathrm{~s}, 1 \mathrm{H}), 8.06(\mathrm{~d}$, $J=7.0 \mathrm{~Hz}, 2 \mathrm{H}), 7.63-7.58(\mathrm{~m}, 3 \mathrm{H}), 7.05(\mathrm{~s}, 1 \mathrm{H}), 7.00(\mathrm{~s}, 1 \mathrm{H}), 5.53-5.32(\mathrm{~m}, 3 \mathrm{H}), 5.26$ $(\mathrm{d}, J=7.6 \mathrm{~Hz}, 1 \mathrm{H}), 4.09(\mathrm{~d}, J=9.6 \mathrm{~Hz}, 1 \mathrm{H}), 3.47-3.37(\mathrm{~m}, 3 \mathrm{H}) .{ }^{13} \mathrm{C}$ NMR $(100 \mathrm{MHz}$, DMSO- $\left.d_{6}\right) \delta 182.99,170.46,164.01,151.74,149.66,147.27,132.48,131.30,131.12$ $129.61 \times 2,126.82 \times 2,106.62,105.22,100.49,94.25,75.99,75.74,73.30,71.79$. LC-MS $m / z$ calcd for $\mathrm{C}_{21} \mathrm{H}_{18} \mathrm{O}_{11} 446.0913$, found $447.0913[\mathrm{M}+\mathrm{H}]^{+}$.

Baicalin ethyl ester (2a). ${ }^{1} \mathrm{H}$ NMR (400 MHz, DMSO- $\left.d_{6}\right) \delta 12.59(\mathrm{~s}, 1 \mathrm{H}), 8.68(\mathrm{~s}$, 1H), $8.06(\mathrm{~d}, J=7.3 \mathrm{~Hz}, 2 \mathrm{H}), 7.63-7.58(\mathrm{~m}, 3 \mathrm{H}), 7.06(\mathrm{~s}, 1 \mathrm{H}), 6.99(\mathrm{~s}, 1 \mathrm{H}), 5.54-5.32$ $(\mathrm{m}, 3 \mathrm{H}), 5.30(\mathrm{~d}, J=7.5 \mathrm{~Hz}, 1 \mathrm{H}), 4.20(\mathrm{~d}, J=9.7 \mathrm{~Hz}, 2 \mathrm{H}), 4.14(\mathrm{~d}, J=9.7 \mathrm{~Hz}, 1 \mathrm{H})$, $3.48-3.35(\mathrm{~m}, 3 \mathrm{H}), 1.22(\mathrm{t}, J=7.0 \mathrm{~Hz}, 3 \mathrm{H}) .{ }^{13} \mathrm{C}$ NMR $\left(100 \mathrm{MHz}\right.$, DMSO- $\left.d_{6}\right) \delta$ $182.99,169.06,164.00,151.70,149.64,147.30,132.49,131.31,131.13,129.59 \times 2$, $126.81 \times 2,106.65,105.24,100.56,94.27,75.86,75.60,73.30,71.78,61.23,14.40$ LC-MS $m / z$ calcd for $\mathrm{C}_{23} \mathrm{H}_{22} \mathrm{O}_{11} 474.1227$, found $475.1227[\mathrm{M}+\mathrm{H}]^{+}$.

Baicalin propyl ester (2b). ${ }^{1} \mathrm{H}$ NMR $\left(400 \mathrm{MHz}, \mathrm{DMSO}-d_{6}\right) \delta 12.58(\mathrm{~s}, 1 \mathrm{H}), 8.67$ (s, 1H), $8.06(\mathrm{~d}, J=7.1 \mathrm{~Hz}, 2 \mathrm{H}), 7.63-7.58(\mathrm{~m}, 3 \mathrm{H}), 7.05(\mathrm{~s}, 1 \mathrm{H}), 7.00(\mathrm{~s}, 1 \mathrm{H})$, 5.54-5.32 (m, 3H), $5.28(\mathrm{~d}, J=7.5 \mathrm{~Hz}, 1 \mathrm{H}), 4.21(\mathrm{~d}, J=9.7 \mathrm{~Hz}, 1 \mathrm{H}), 4.09-4.05(\mathrm{~m}$, 2H), 3.49-3.34 (m, 3H), $1.61(\mathrm{q}, J=7.0 \mathrm{~Hz}, 2 \mathrm{H}), 0.88(\mathrm{t}, J=7.4 \mathrm{~Hz}, 3 \mathrm{H}) .{ }^{13} \mathrm{C}$ NMR $\left(100 \mathrm{MHz}, \mathrm{DMSO}-d_{6}\right) \delta 182.99,169.07,163.98,151.67,149.61,147.27,132.51$, $131.30,131.13,129.58 \times 2,126.79 \times 2,106.66,105.25,100.70,94.35,75.81,75.63$, 73.32, 71.70, 66.57, 21.87, 10.58. LC-MS $m / z$ calcd for $\mathrm{C}_{24} \mathrm{H}_{24} \mathrm{O}_{11} 488.1385$, found $489.1385[\mathrm{M}+\mathrm{H}]^{+}$. 
Baicalin butyl ester (2c). ${ }^{1} \mathrm{H}$ NMR (400 MHz, DMSO- $\left.d_{6}\right) \delta 12.59(\mathrm{~s}, 1 \mathrm{H}), 8.69$ (s, $1 \mathrm{H}), 8.07(\mathrm{~d}, J=7.0 \mathrm{~Hz}, 2 \mathrm{H}), 7.63-7.58(\mathrm{~m}, 3 \mathrm{H}), 7.04(\mathrm{~s}, 1 \mathrm{H}), 7.01(\mathrm{~s}, 1 \mathrm{H}), 5.55-5.33$ $(\mathrm{m}, 3 \mathrm{H}), 5.29(\mathrm{~d}, J=7.6 \mathrm{~Hz}, 1 \mathrm{H}), 4.21(\mathrm{~d}, J=9.7 \mathrm{~Hz}, 1 \mathrm{H}), 4.08(\mathrm{~d}, J=6.4 \mathrm{~Hz}, 2 \mathrm{H})$, 3.49-3.35 (m, 3H), 1.58-1.54 (m, 2H), 1.32 (q, $J=7.5 \mathrm{~Hz}, 2 \mathrm{H}), 0.80$ (t, $J=7.4 \mathrm{~Hz}$, 3H). ${ }^{13} \mathrm{C}$ NMR (100 MHz, DMSO- $\left.d_{6}\right) \delta 182.99,169.06,163.98,151.66,149.61$, $147.26,132.51,131.31,131.11,129.58 \times 2,126.78 \times 2,106.63,105.24,100.58$ $94.31,75.78,75.62,73.30,71.68,64.81,30.47,18.91,13.89$. LC-MS $m / z$ calcd for $\mathrm{C}_{25} \mathrm{H}_{26} \mathrm{O}_{11}$ 502.1553, found 503.1553 [M+ H] $]^{+}$.

Baicalin hexyl ester (2d). ${ }^{1} \mathrm{H}$ NMR (400 MHz, DMSO- $\left.d_{6}\right) \delta 12.59$ (s, $\left.1 \mathrm{H}\right), 8.68$ (s, 1H), $8.07(\mathrm{~d}, J=7.1 \mathrm{~Hz}, 2 \mathrm{H}), 7.64-7.58(\mathrm{~m}, 3 \mathrm{H}), 7.04(\mathrm{~s}, 1 \mathrm{H}), 7.02(\mathrm{~s}, 1 \mathrm{H}), 5.54-5.32$ $(\mathrm{m}, 3 \mathrm{H}), 5.30(\mathrm{~d}, J=7.5 \mathrm{~Hz}, 1 \mathrm{H}), 4.21(\mathrm{~d}, J=9.7 \mathrm{~Hz}, 1 \mathrm{H}), 4.07-4.03(\mathrm{~m}, 2 \mathrm{H})$, $3.48-3.35(\mathrm{~m}, 3 \mathrm{H}), 1.58-1.53(\mathrm{~m}, 2 \mathrm{H}), 1.28-1.09(\mathrm{~m}, 6 \mathrm{H}), 0.71(\mathrm{t}, J=7.0 \mathrm{~Hz}, 3 \mathrm{H}) .{ }^{13} \mathrm{C}$ NMR (100 MHz, DMSO- $\left.d_{6}\right) \delta 182.98,169.03,163.97,151.65,149.63,147.25$, $132.50,131.30,131.09,129.58 \times 2,126.77 \times 2,106.61,105.22,100.49,94.26,75.77$, $75.62,73.29,71.63,65.04,31.21,28.41,25.29,22.34,14.18$. LC-MS $m / z$ calcd for $\mathrm{C}_{27} \mathrm{H}_{30} \mathrm{O}_{11} 530.1856$, found $531.1856[\mathrm{M}+\mathrm{H}]^{+}$.

Baicalin octyl ester (2e). ${ }^{1} \mathrm{H}$ NMR (400 MHz, DMSO- $\left.d_{6}\right) \delta 12.58(\mathrm{~s}, 1 \mathrm{H}), 8.67(\mathrm{~s}$, 1H), $8.07(\mathrm{~d}, J=6.9 \mathrm{~Hz}, 2 \mathrm{H}), 7.64-7.58(\mathrm{~m}, 3 \mathrm{H}), 7.04(\mathrm{~s}, 1 \mathrm{H}), 7.02(\mathrm{~s}, 1 \mathrm{H}), 5.54-5.32$ (m, 3H), $5.29(\mathrm{~d}, J=7.5 \mathrm{~Hz}, 1 \mathrm{H}), 4.21(\mathrm{~d}, J=9.7 \mathrm{~Hz}, 1 \mathrm{H}), 4.07-4.03(\mathrm{~m}, 2 \mathrm{H})$, 3.51-3.34 (m, 3H), 1.58-1.53 (m, 2H), 1.41-0.90 (m, 10H), $0.75(\mathrm{t}, J=7.2 \mathrm{~Hz}, 3 \mathrm{H})$. ${ }^{13} \mathrm{C}$ NMR $\left(100 \mathrm{MHz}, \mathrm{DMSO}-d_{6}\right) \delta 182.99,168.99,163.94,151.64,149.61,147.24$, $132.50,131.28,131.07,129.58 \times 2,126.75 \times 2,106.61,105.20,100.55,94.24,75.74$ 
$75.60,73.29,71.57,65.01,31.60,29.01,28.99,28.47,25.65,22.45,14.29$. LC-MS $m / z$ calcd for $\mathrm{C}_{29} \mathrm{H}_{34} \mathrm{O}_{11} 558.2179$, found $559.2179[\mathrm{M}+\mathrm{H}]^{+}$.

Baicalin geranyl ester (2f). ${ }^{1} \mathrm{H}$ NMR $\left(400 \mathrm{MHz}, \mathrm{DMSO}-d_{6}\right) \delta 12.58(\mathrm{~s}, 1 \mathrm{H}), 8.72$ $(\mathrm{s}, 1 \mathrm{H}), 8.03(\mathrm{~d}, J=7.1 \mathrm{~Hz}, 2 \mathrm{H}), 7.61-7.54(\mathrm{~m}, 3 \mathrm{H}), 7.02(\mathrm{~s}, 1 \mathrm{H}), 6.96(\mathrm{~s}, 1 \mathrm{H})$, 5.52-5.30 (m, 3H), $5.37(\mathrm{~s}, 1 \mathrm{H}), 5.27(\mathrm{~d}, J=6.2 \mathrm{~Hz}, 1 \mathrm{H}), 4.93(\mathrm{~s}, 1 \mathrm{H}), 4.64(\mathrm{dd}, J=$ $12.5,7.0 \mathrm{~Hz}, 2 \mathrm{H}), 4.21(\mathrm{~d}, J=9.7 \mathrm{~Hz}, 1 \mathrm{H}), 3.51-3.39(\mathrm{~m}, 3 \mathrm{H}), 1.92-1.87(\mathrm{~m}, 4 \mathrm{H})$, $1.60(\mathrm{~s}, 3 \mathrm{H}), 1.54(\mathrm{~s}, 3 \mathrm{H}), 1.44(\mathrm{~s}, 3 \mathrm{H}) .{ }^{13} \mathrm{C}$ NMR $\left(100 \mathrm{MHz}, \mathrm{DMSO}-d_{6}\right) \delta 182.94$, $168.98,163.98,151.63,149.61,147.26,142.42,132.43,131.43,131.24,131.10$ $129.53 \times 2,126.72 \times 2,124.01,118.39,106.62,105.16,100.51,94.22,75.83,75.57$, $73.29,71.75,61.87,39.28,26.17,25.79,17.83,16.63$. LC-MS $\mathrm{m} / \mathrm{z}$ calcd for $\mathrm{C}_{31} \mathrm{H}_{34} \mathrm{O}_{11}$ 582.2173, found 583.2173 [M $\left.+\mathrm{H}\right]^{+}$.

Baicalin citronellyl ester (2g). ${ }^{1} \mathrm{H}$ NMR (400 MHz, DMSO- $\left.d_{6}\right) \delta 12.57(\mathrm{~s}, 1 \mathrm{H})$, $8.72(\mathrm{~s}, 1 \mathrm{H}), 8.03(\mathrm{~d}, J=7.5 \mathrm{~Hz}, 2 \mathrm{H}), 7.60-7.54(\mathrm{~m}, 3 \mathrm{H}), 7.01(\mathrm{~s}, 1 \mathrm{H}), 6.97(\mathrm{~s}, 1 \mathrm{H})$, 5.59-5.37 (m, 3H), $5.29(\mathrm{~d}, J=7.3 \mathrm{~Hz}, 1 \mathrm{H}), 4.89(\mathrm{~d}, J=7.0 \mathrm{~Hz}, 1 \mathrm{H}), 4.20(\mathrm{~d}, J=6.8$ $\mathrm{Hz}, 1 \mathrm{H}), 4.07$ (ddt, $J=18.2,12.2,6.7 \mathrm{~Hz}, 2 \mathrm{H}), 3.51-3.45(\mathrm{~m}, 3 \mathrm{H}), 1.81-1.71(\mathrm{~m}, 2 \mathrm{H})$, 1.62-0.95 (m, 5H), $1.53(\mathrm{~d}, J=5.1 \mathrm{~Hz}, 3 \mathrm{H}), 1.41(\mathrm{~d}, J=19.3 \mathrm{~Hz}, 3 \mathrm{H}), 0.77(\mathrm{t}, J=7.0$ $\mathrm{Hz}, 3 \mathrm{H}) .{ }^{13} \mathrm{C}$ NMR $\left(100 \mathrm{MHz}, \mathrm{DMSO}-d_{6}\right) \delta 182.95,169.01,163.96,151.59,149.60$, $147.23,132.45,131.23,131.10,130.85,129.53 \times 2,126.71 \times 2,124.81,106.61$, $105.15,100.59,94.26,75.80,75.56,73.27,71.64,63.48,36.87,35.25,29.28,25.82$, 25.22, 19.58, 17.78. LC-MS $m / z$ calcd for $\mathrm{C}_{31} \mathrm{H}_{36} \mathrm{O}_{11}$ 584.2338, found 585.2338 [M+ $\mathrm{H}]^{+}$.

Baicalin decyl ester (2h). ${ }^{1} \mathrm{H}$ NMR (400 MHz, DMSO- $\left.d_{6}\right) \delta 12.56(\mathrm{~s}, 1 \mathrm{H}), 8.71(\mathrm{~s}$, 
1H), $8.03(\mathrm{~d}, J=7.3 \mathrm{~Hz}, 2 \mathrm{H}), 7.61-7.55(\mathrm{~m}, 3 \mathrm{H}), 7.01(\mathrm{~s}, 1 \mathrm{H}), 6.98(\mathrm{~s}, 1 \mathrm{H}), 5.50-5.34$ (m, 3H), 5.27 (d, $J=7.4 \mathrm{~Hz}, 1 \mathrm{H}), 4.21(\mathrm{~d}, J=9.5 \mathrm{~Hz}, 1 \mathrm{H}), 4.05-4.01(\mathrm{~m}, 2 \mathrm{H})$, 3.52-3.40 (m, 3H), 1.56-1.51 (m, 2H), 1.24-0.99 (m, 14H), 0.77 (t, $J=7.3 \mathrm{~Hz}, 3 \mathrm{H})$. ${ }^{13} \mathrm{C}$ NMR (100 MHz, DMSO- $\left.d_{6}\right) \delta 182.95,168.96,163.91,151.64,149.58,147.23$, $132.46,131.19,131.08,129.54 \times 2,126.62 \times 2,106.64,105.11,100.64,94.21,75.73$, $75.56,73.27,71.54,65.01,31.67,29.34 \times 2,29.09,29.03,28.45,25.63,22.50,14.32$. LC-MS $m / z$ calcd for $\mathrm{C}_{31} \mathrm{H}_{38} \mathrm{O}_{11} 586.1539$, found $587.1539[\mathrm{M}+\mathrm{H}]^{+}$.

Baicalin undec-10-en-1-yl ester (2i). ${ }^{1} \mathrm{H}$ NMR (400 MHz, DMSO- $\left.d_{6}\right) \delta 12.59$ (s, 1H), $8.63(\mathrm{~s}, 1 \mathrm{H}), 8.05(\mathrm{~d}, J=6.7 \mathrm{~Hz}, 2 \mathrm{H}), 7.58-7.54(\mathrm{~m}, 3 \mathrm{H}), 6.99(\mathrm{~s}, 1 \mathrm{H}), 6.96$ (s, 1H), $5.76(\mathrm{ddt}, J=17.1,10.3,6.7 \mathrm{~Hz}, 1 \mathrm{H}), 5.48-5.34(\mathrm{~m}, 3 \mathrm{H}), 5.23(\mathrm{~d}, J=7.6 \mathrm{~Hz}$, 1H), $4.96(\mathrm{ddd}, J=17.1,3.7,1.5 \mathrm{~Hz}, 1 \mathrm{H}), 4.93-4.88(\mathrm{~m}, 1 \mathrm{H}), 4.19(\mathrm{~d}, J=9.7 \mathrm{~Hz}$, $1 \mathrm{H}), 4.19(\mathrm{~d}, J=9.7 \mathrm{~Hz}, 2 \mathrm{H}), 3.49-3.38(\mathrm{~m}, 3 \mathrm{H}), 2.01-1.93(\mathrm{~m}, 2 \mathrm{H}), 1.70(\mathrm{~s}, 1 \mathrm{H})$, $1.64(\mathrm{~s}, 1 \mathrm{H}), 1.41(\mathrm{p}, J=6.7 \mathrm{~Hz}, 2 \mathrm{H}), 1.35-1.24(\mathrm{~m}, 10 \mathrm{H}) .{ }^{13} \mathrm{C}$ NMR (100 MHz, DMSO- $\left.d_{6}\right) \delta 182.91,168.82,163.83,151.59,149.65,147.34,139.00,132.16,131.32$ $130.98,129.36 \times 2,126.61 \times 2,114.60,106.73,105.09,101.05,94.23,75.82,75.53$, $73.29,71.58,61.26,33.71,33.06,29.65,29.54,29.45,29.10,28.87,26.04$. LC-MS $m / z$ calcd for $\mathrm{C}_{32} \mathrm{H}_{38} \mathrm{O}_{11} 598.2490$, found $599.2490[\mathrm{M}+\mathrm{H}]^{+}$.

Baicalin undecanyl ester (2j). ${ }^{1} \mathrm{H}$ NMR $\left(400 \mathrm{MHz}, \mathrm{DMSO}-d_{6}\right) \delta 12.58(\mathrm{~s}, 1 \mathrm{H})$, $8.64(\mathrm{~s}, 1 \mathrm{H}), 8.05(\mathrm{~d}, J=1.8 \mathrm{~Hz}, 2 \mathrm{H}), 7.60-7.56(\mathrm{~m}, 3 \mathrm{H}), 7.01(\mathrm{~s}, 1 \mathrm{H}), 6.99(\mathrm{~s}, 1 \mathrm{H})$, 5.48-5.33 (m, 3H), $5.24(\mathrm{~d}, J=7.3 \mathrm{~Hz}, 1 \mathrm{H}), 4.19(\mathrm{~d}, J=9.7 \mathrm{~Hz}, 1 \mathrm{H}), 4.08-4.03(\mathrm{~m}$, $2 \mathrm{H}), 3.52-3.36(\mathrm{~m}, 3 \mathrm{H}), 1.70(\mathrm{~s}, 1 \mathrm{H}), 1.64(\mathrm{~s}, 1 \mathrm{H}), 1.58-1.56(\mathrm{~m}, 2 \mathrm{H}), 1.42-1.38(\mathrm{~m}$, $2 \mathrm{H}), 1.27-1.21(\mathrm{~m}, 12 \mathrm{H}), 0.86-0.82(\mathrm{~m}, 3 \mathrm{H}) .{ }^{13} \mathrm{C}$ NMR (100 MHz, DMSO- $\left.d_{6}\right) \delta$ 
$182.94,168.88,163.86,151.62,149.57,147.30,132.28,131.31,131.18,129.44 \times 2$, $126.66 \times 2,106.68,105.13,100.88,94.23,75.78,75.55,73.29,71.57,61.22,33.06$, $31.83,29.66,29.60,29.57,29.53,29.26,26.03,22.59,14.24$. LC-MS $\mathrm{m} / z$ calcd for $\mathrm{C}_{32} \mathrm{H}_{40} \mathrm{O}_{11}$ 600.2617, found $601.2617[\mathrm{M}+\mathrm{H}]^{+}$.

Baicalin lauryl ester (2k). ${ }^{1} \mathrm{H}$ NMR $\left(400 \mathrm{MHz}, \mathrm{DMSO}-d_{6}\right) \delta 12.58(\mathrm{~s}, 1 \mathrm{H}), 8.62$ (s, 1H), $8.02(\mathrm{~d}, J=8.2 \mathrm{~Hz}, 2 \mathrm{H}), 7.52(\mathrm{~s}, 3 \mathrm{H}), 6.97(\mathrm{~s}, 1 \mathrm{H}), 6.90(\mathrm{~s}, 1 \mathrm{H}), 5.47-5.36$ $(\mathrm{m}, 3 \mathrm{H}), 5.21(\mathrm{~d}, J=7.5 \mathrm{~Hz}, 1 \mathrm{H}), 4.34(\mathrm{~s}, 1 \mathrm{H}), 4.18(\mathrm{~d}, J=9.7 \mathrm{~Hz}, 1 \mathrm{H}), 3.95(\mathrm{t}, J=$ $6.7 \mathrm{~Hz}, 1 \mathrm{H}), 3.51-3.38(\mathrm{~m}, 3 \mathrm{H}), 1.94(\mathrm{~s}, 1 \mathrm{H}), 1.62-1.54(\mathrm{~m}, 1 \mathrm{H}), 1.41(\mathrm{p}, J=6.8 \mathrm{~Hz}$ $6 \mathrm{H}), 1.30-1.23(\mathrm{~m}, 12 \mathrm{H}), 0.84(\mathrm{t}, J=7.1 \mathrm{~Hz}, 3 \mathrm{H}) .{ }^{13} \mathrm{C}$ NMR $\left(100 \mathrm{MHz}, \mathrm{DMSO}-d_{6}\right) \delta$ $182.84,168.78,163.75,151.54,149.53,147.36,131.90,131.37,131.28,129.20 \times 2$, $126.53 \times 2,106.75,105.03,101.18,94.24,75.81,75.50,73.28,71.59,61.30,33.07$, $31.93,29.80,29.75,29.74,29.70,29.65,29.38,26.09,22.63,14.03$. LC-MS $m / z$ calcd for $\mathrm{C}_{33} \mathrm{H}_{42} \mathrm{O}_{11}$ 614.2811, found 615.2811 [M + H] $]^{+}$. 
Table S1. The MBC values of baicalin and its ester derivatives

\begin{tabular}{|c|c|c|c|c|}
\hline \multirow{2}{*}{ compd } & \multirow{2}{*}{$\mathrm{Clog} P^{\alpha}$} & \multicolumn{3}{|c|}{$\mathrm{MBC}(\mathrm{mM})$} \\
\hline & & S. aureus & E.coli & C. albicans \\
\hline 1 & 0.8 & $>12.8$ & $>12.8$ & $>12.8$ \\
\hline $2 a$ & 1.7 & $>12.8$ & $>12.8$ & $>12.8$ \\
\hline $2 b$ & 2.2 & $>12.8$ & $>12.8$ & $>12.8$ \\
\hline $2 \mathrm{c}$ & 2.8 & $>12.8$ & $>12.8$ & 12.8 \\
\hline 2d & 3.8 & $>12.8$ & $>12.8$ & 12.8 \\
\hline $2 e$ & 4.9 & 12.8 & $>12.8$ & 6.4 \\
\hline $2 f$ & 4.9 & 12.8 & $>12.8$ & 3.2 \\
\hline $2 \mathrm{~g}$ & 5.2 & 6.4 & 12.8 & 1.6 \\
\hline $2 \mathrm{~h}$ & 5.9 & $>12.8$ & $>12.8$ & $>12.8$ \\
\hline $2 \mathbf{i}$ & 6.0 & 12.8 & $>12.8$ & 12.8 \\
\hline $2 j$ & 6.5 & $>12.8$ & $>12.8$ & $>12.8$ \\
\hline $2 \mathbf{k}$ & 7.0 & $>12.8$ & $>12.8$ & $>12.8$ \\
\hline
\end{tabular}

${ }^{\alpha}$ The partition coefficient $(\log P)$ values of baicalin and its ester derivatives were calculated by the ChemBioDraw Ultra 14.0 software (PerkinElmer, USA). 
Table S2. Comparison of MIC values of physical mixture of baicalin and geraniol/citronellol and their esters

\begin{tabular}{cccc}
\hline \multirow{2}{*}{ compd } & \multicolumn{3}{c}{ MIC $(\mathrm{mM})$} \\
\cline { 2 - 4 } & S. aureus & E.coli & C. albicans \\
\hline baicalin geranyl ester & 1.6 & 3.2 & 0.8 \\
BAI + geraniol & 6.4 & $>12.8$ & 3.2 \\
baicalin citronellyl ester & 0.8 & 3.2 & 0.4 \\
BAI + citronellol & 6.4 & 12.8 & 3.2 \\
\hline
\end{tabular}



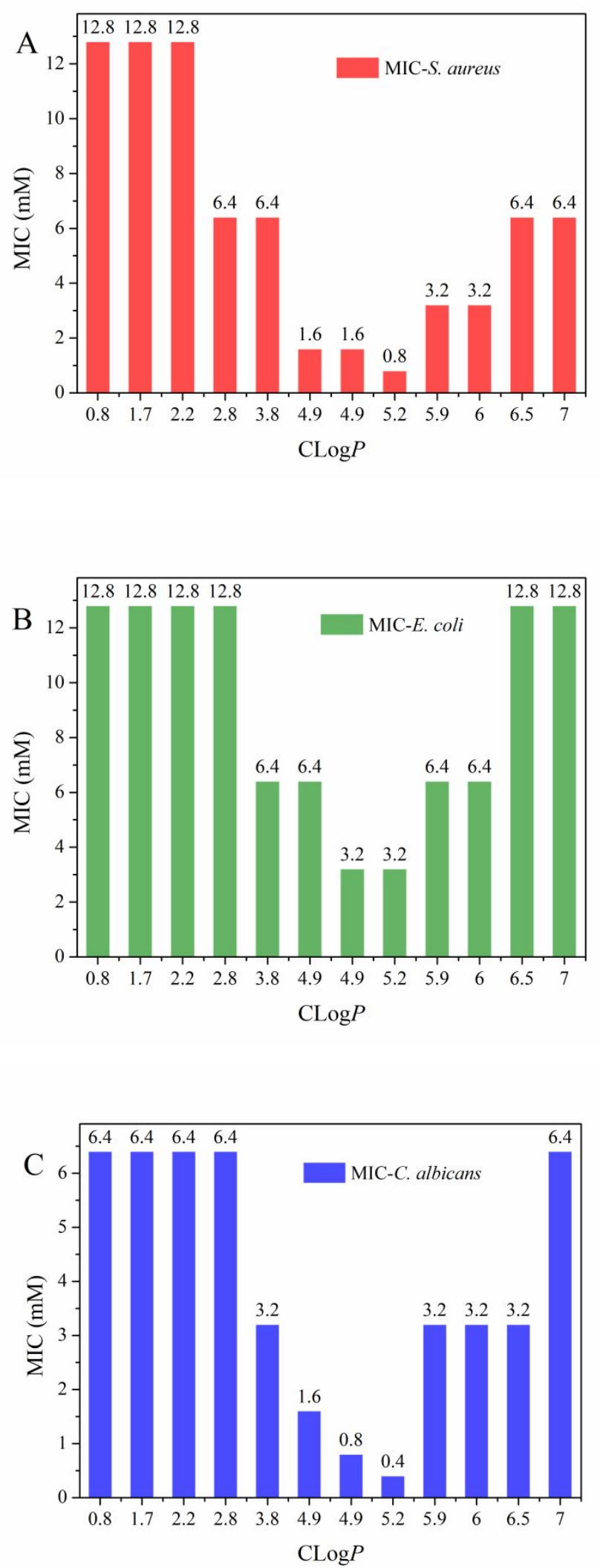

Figure S1. Correlation of antimicrobial activity (MIC) against S. aureus (A), E. coli (B) and C. albicans (C) with CLogP of compounds $\mathbf{1}, \mathbf{2 a - 2} \mathbf{k}$. 


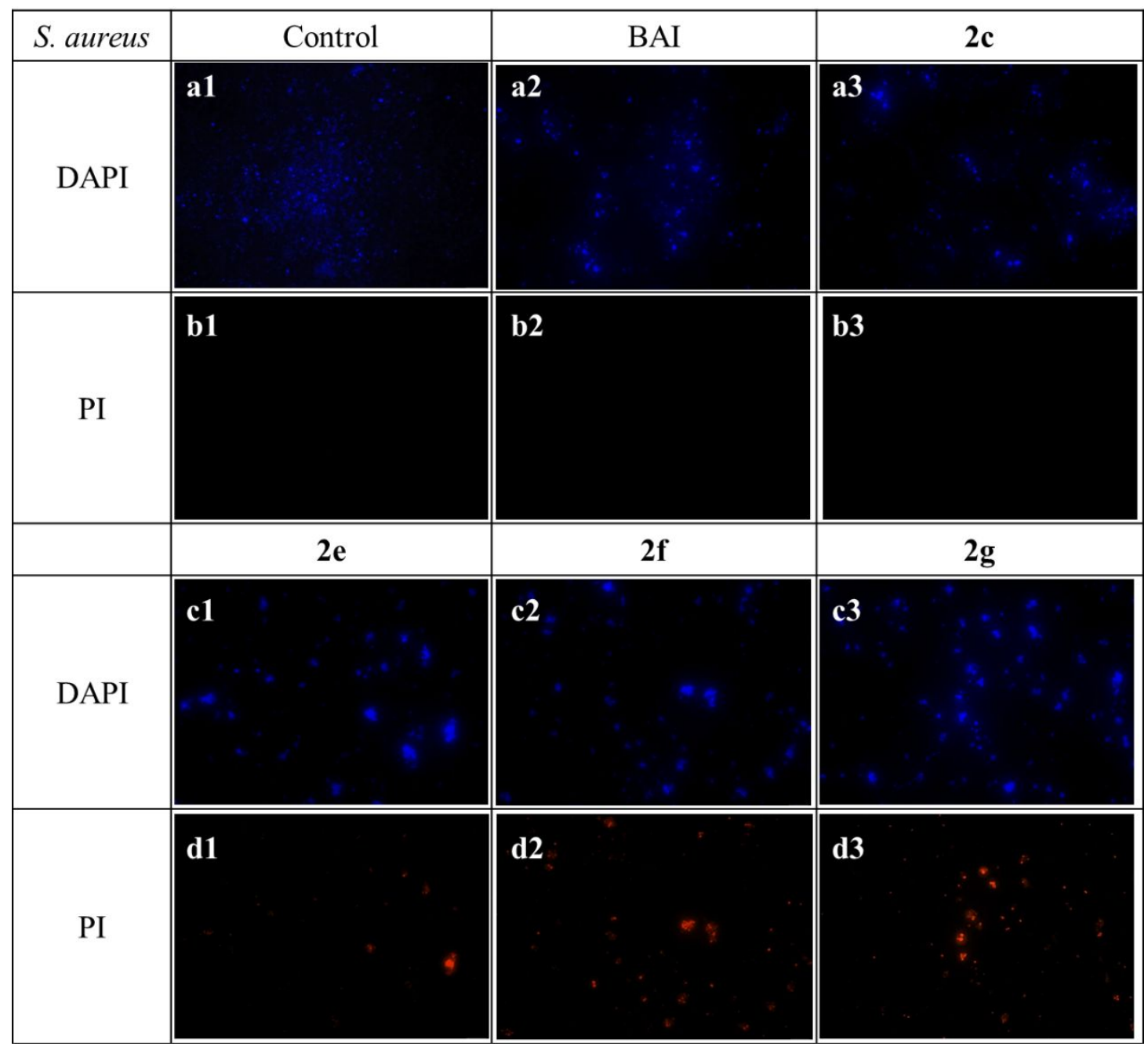

Figure S2. Fluorescence micrographs of $S$. aureus with or without baicalin and its esters. (a1) Control, no treatment, DAPI stained; (b1) Control, no treatment, PI stained; (a2, a3, c1-c3) BAI/2c/2e/2f/2g treatment, DAPI stained; (b2, b3, d1-d3) $\mathrm{BAI} / \mathbf{2 c} / \mathbf{2 e} / \mathbf{2 f} / \mathbf{2 g}$ treatment, PI stained. Scale bar $=20 \mu \mathrm{m}$. 


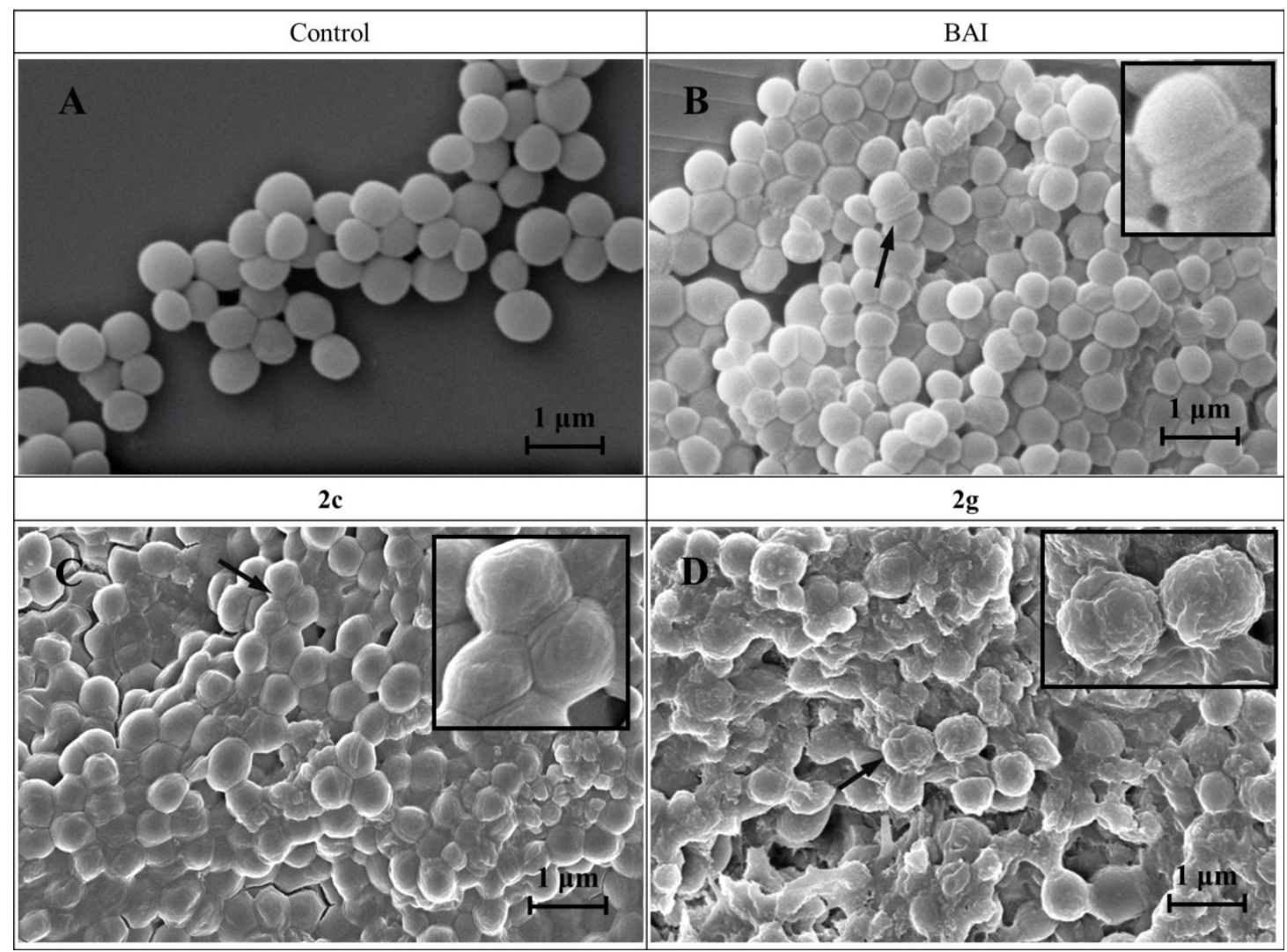

Figure S3. SEM analysis of $S$. aureus with or without baicalin and its esters. (A)

Control, no treatment; (B) treatment with BAI; (C) treatment with $2 \mathbf{c}$; (D) treatment with 2 g. 
HPLC chromatography, ${ }^{1} \mathrm{H}$ NMR and ${ }^{13} \mathrm{C}$ NMR spectra of compounds $1,2 \mathrm{a}-2 \mathrm{k}$<smiles>O=c1cc(-c2ccccc2)oc2cc(O)c(O)c(O)c12</smiles>

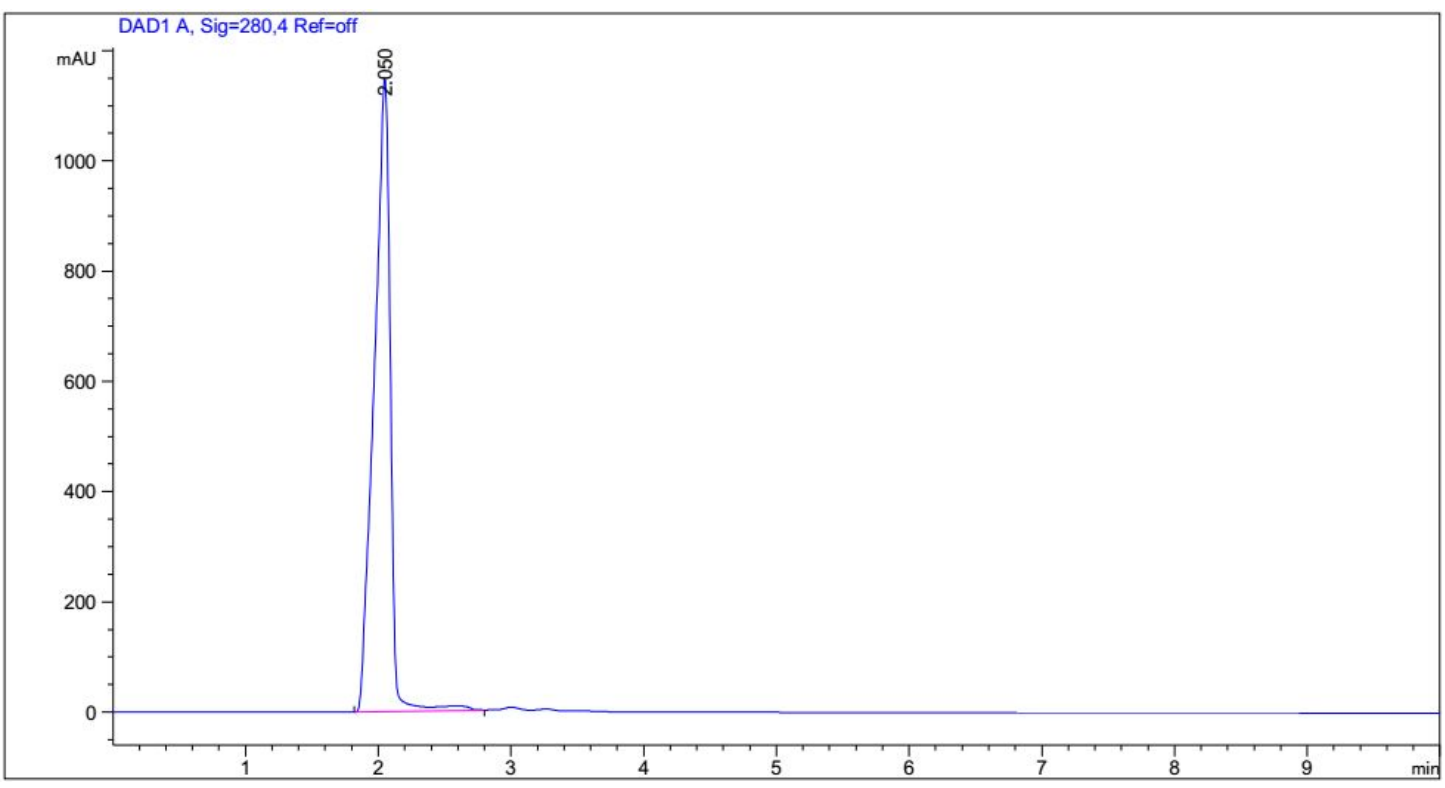

Figure S4. HPLC chromatography result of compound 1

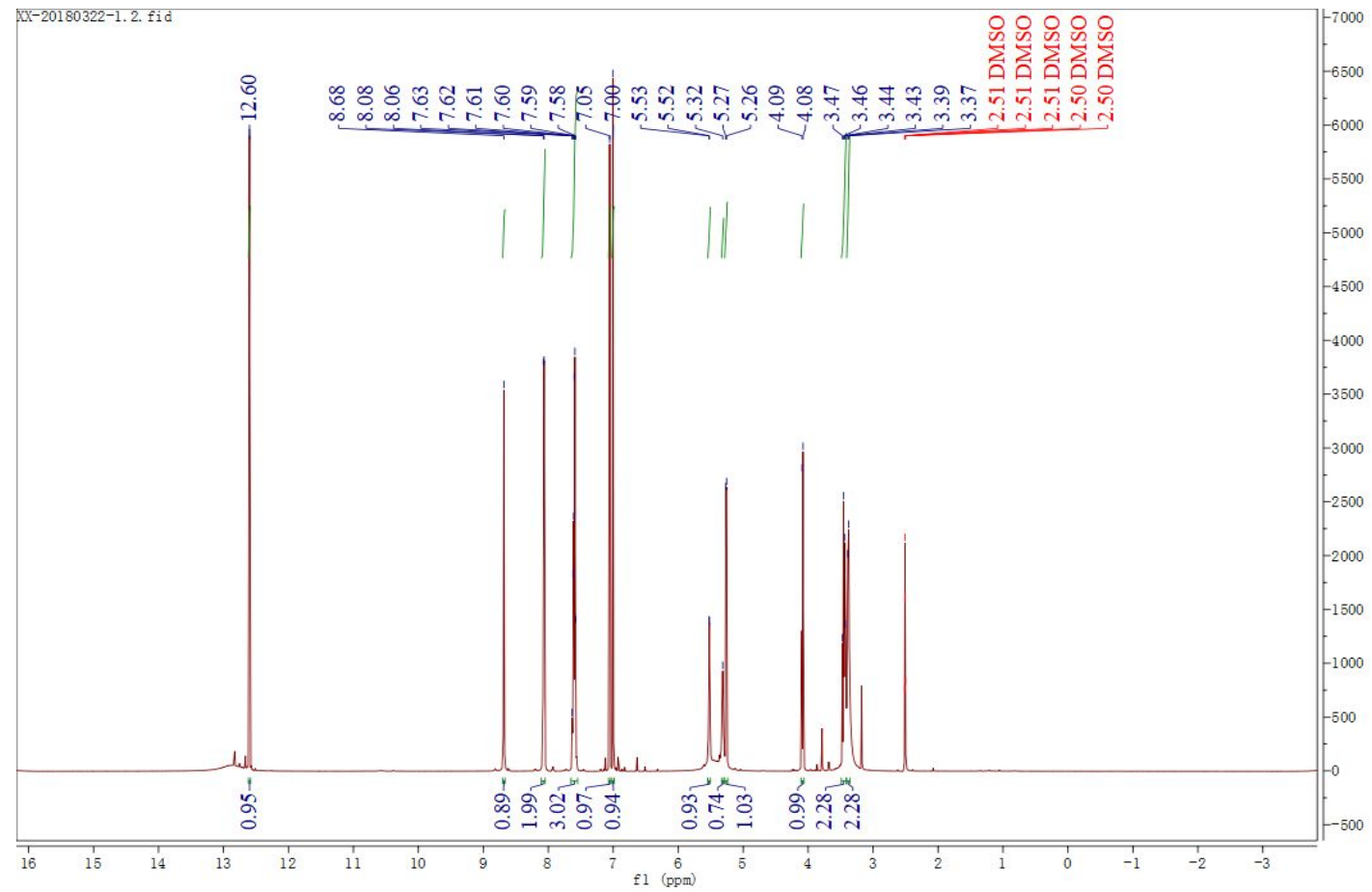

Figure S5. ${ }^{1} \mathrm{H}$ NMR spectrum for compound 1 in DMSO- $d_{6}(400 \mathrm{MHz})$. 


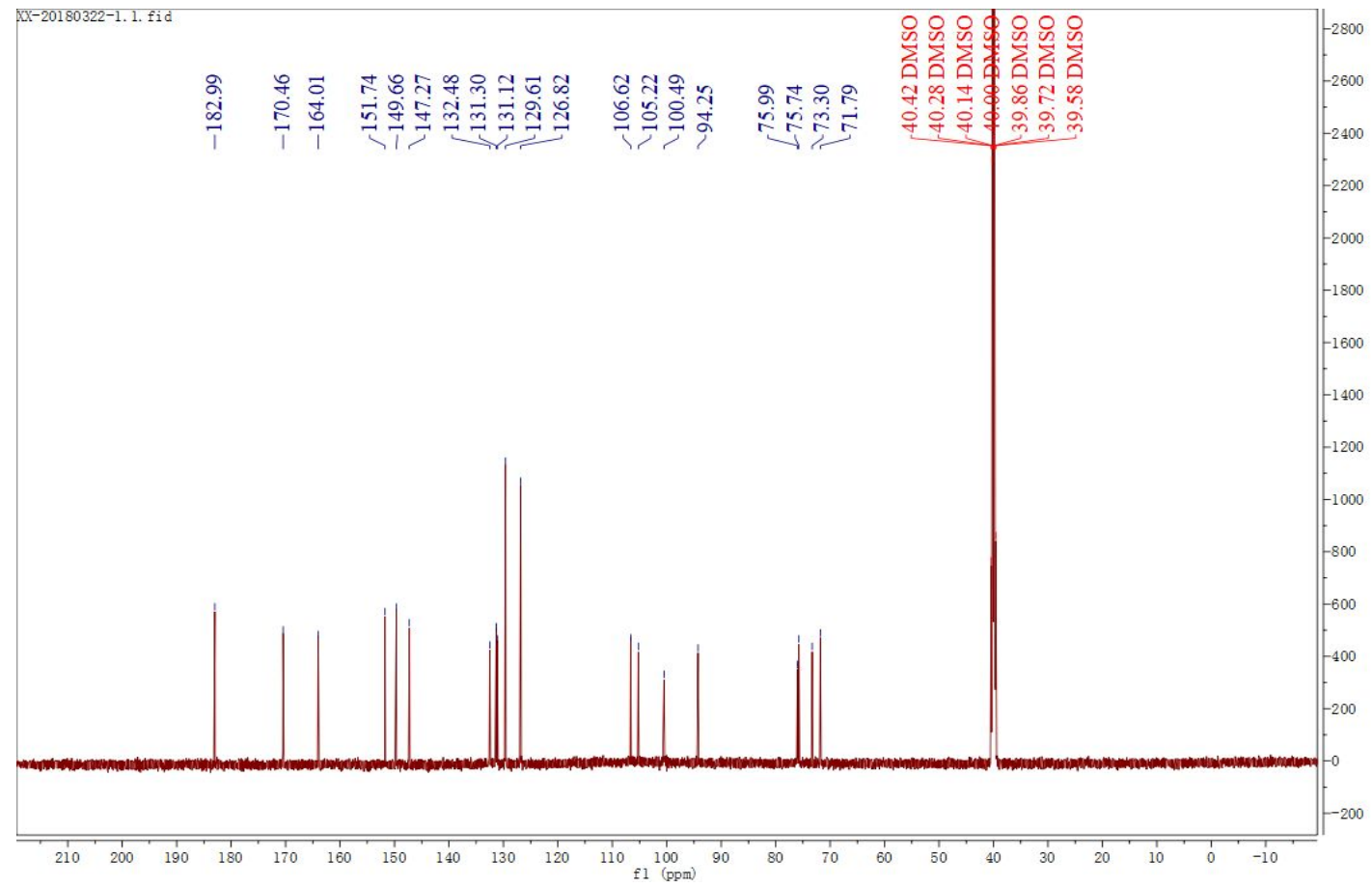

Figure S6. ${ }^{13} \mathrm{C}$ NMR spectrum for compound 1 in DMSO- $d_{6}(100 \mathrm{MHz})$. 

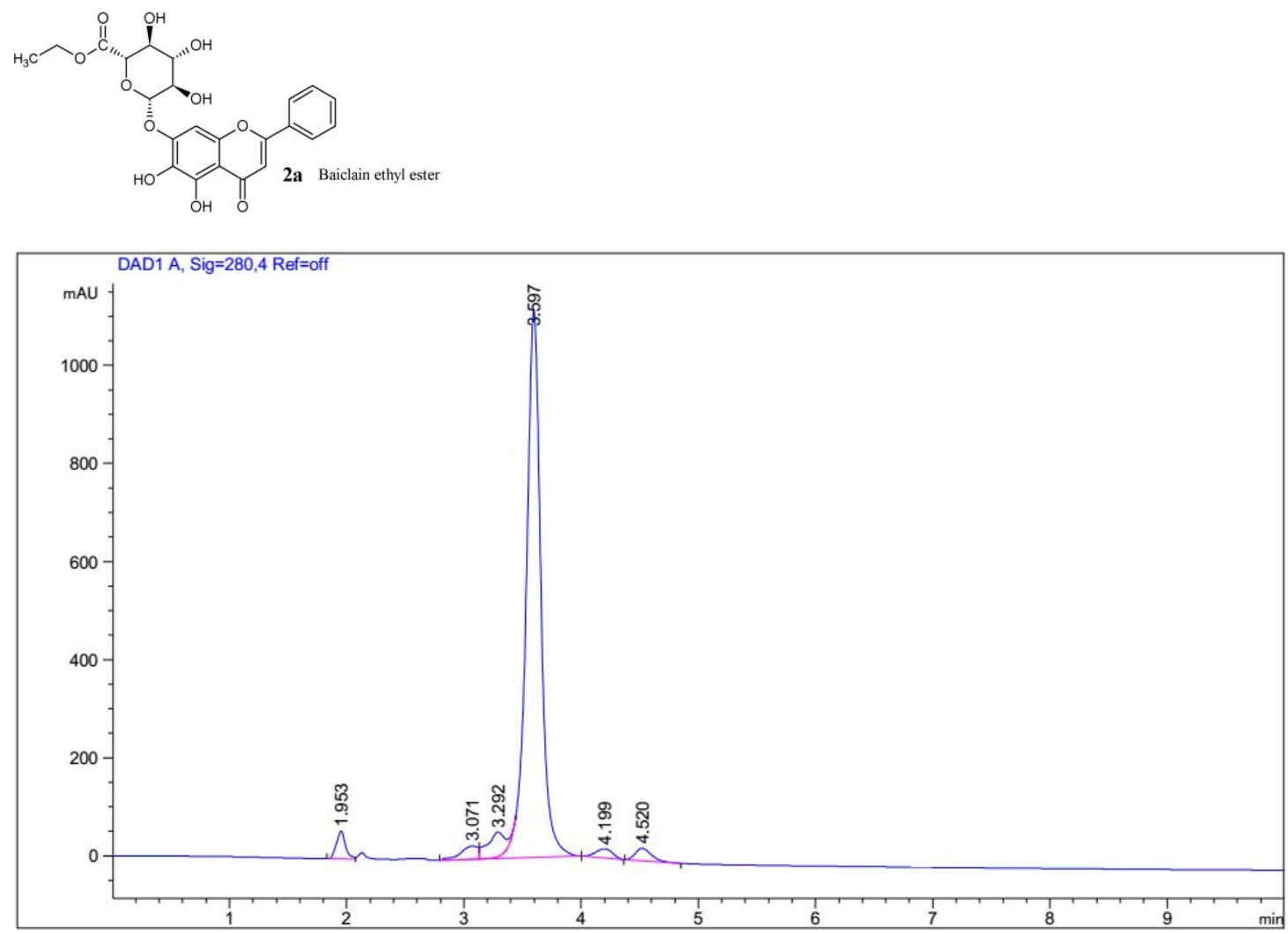

Figure S7. HPLC chromatography result of compound 2a

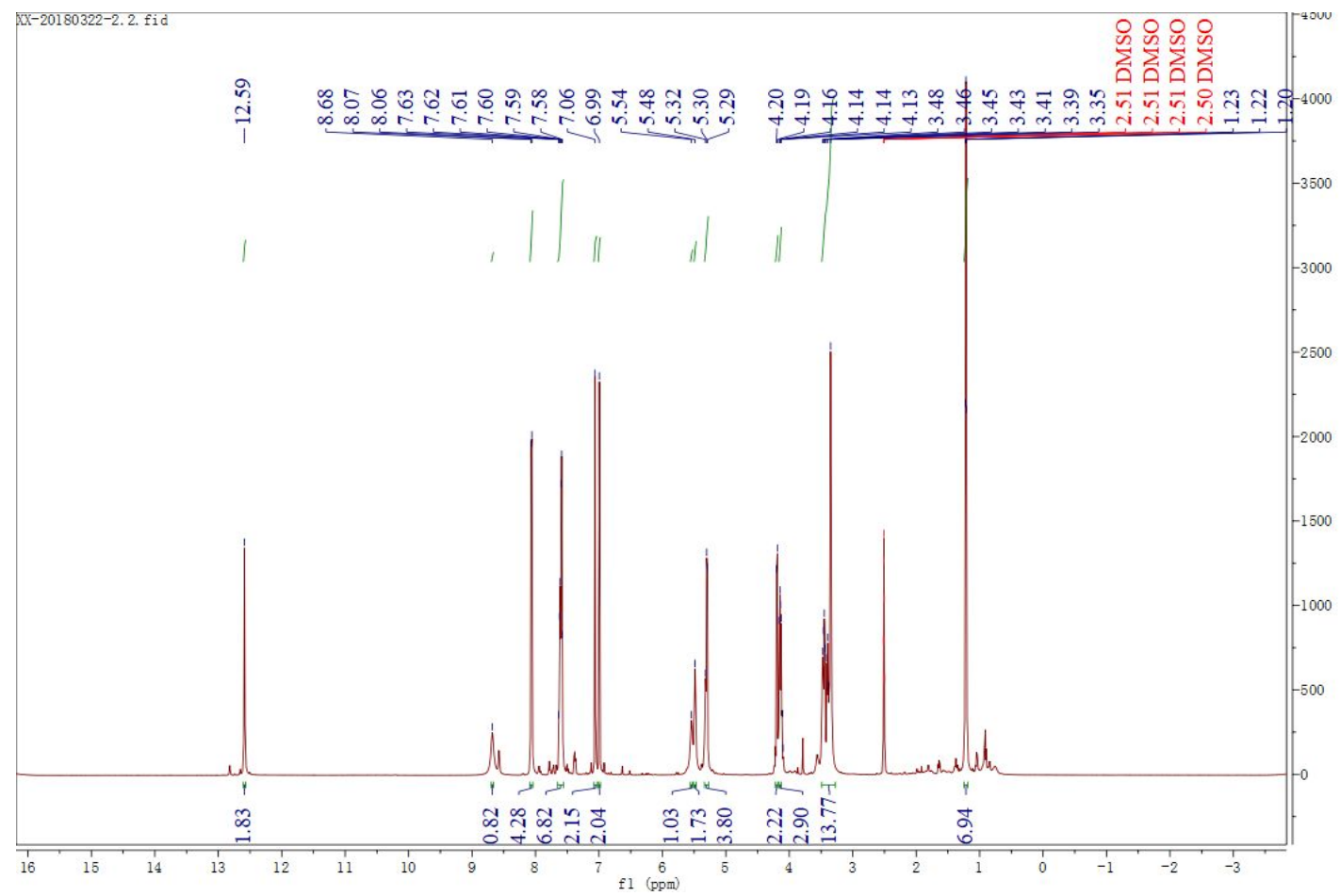

Figure S8. ${ }^{1} \mathrm{H}$ NMR spectrum for compound 2a in DMSO- $d_{6}(400 \mathrm{MHz})$. 


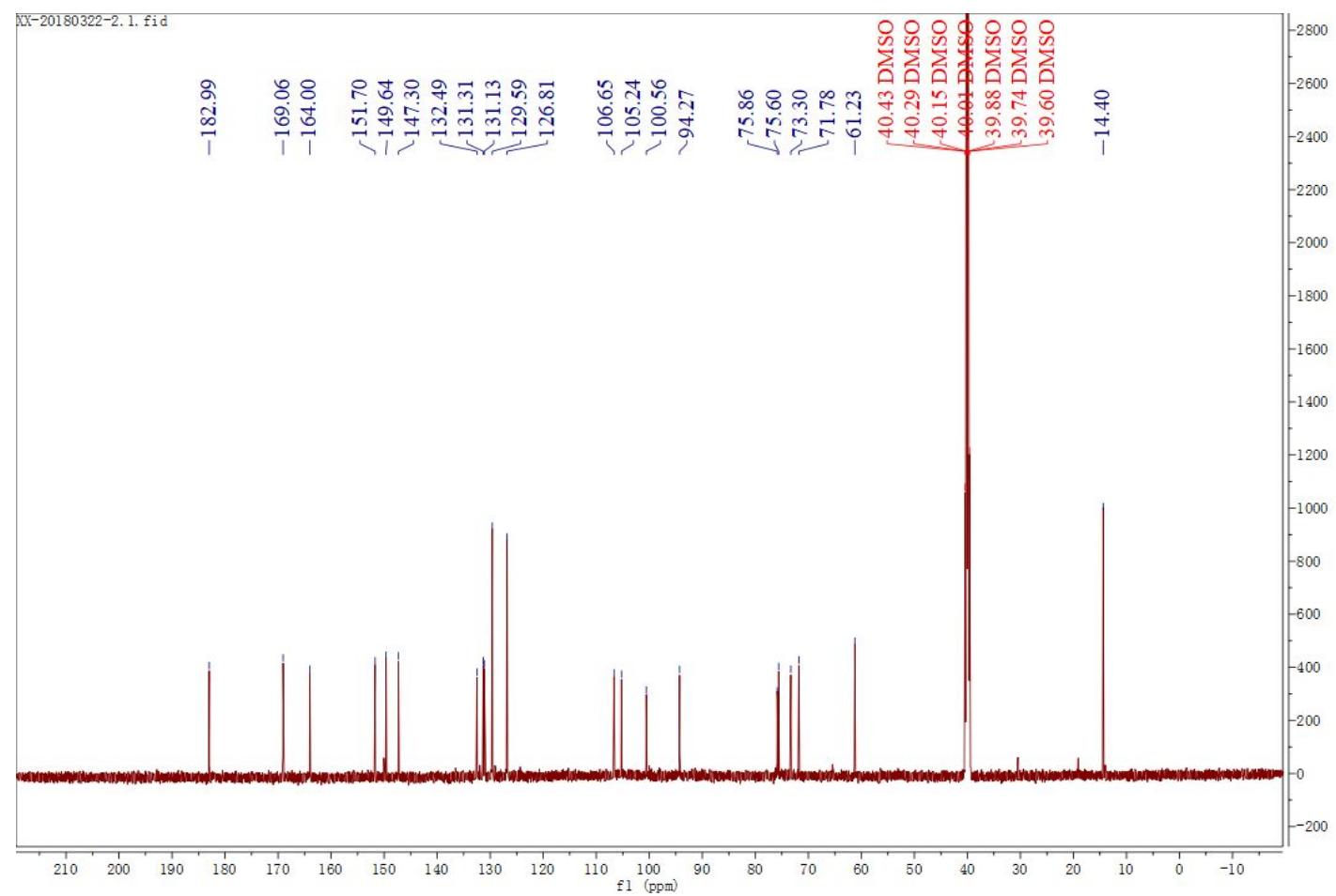

Figure S9. ${ }^{13} \mathrm{C}$ NMR spectrum for compound 2a in DMSO- $d_{6}(100 \mathrm{MHz})$. 

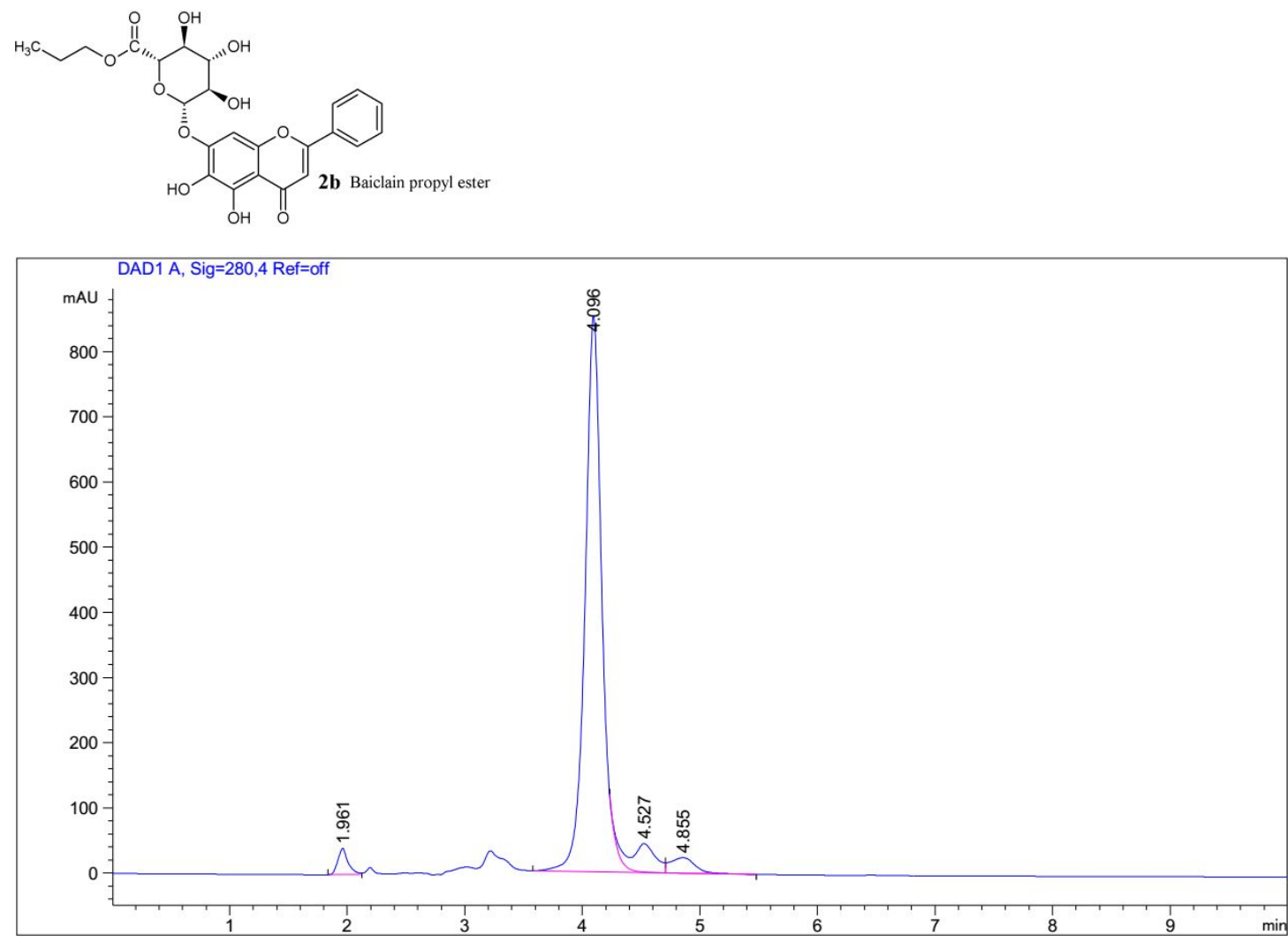

Figure S10. HPLC chromatography result of compound 2b

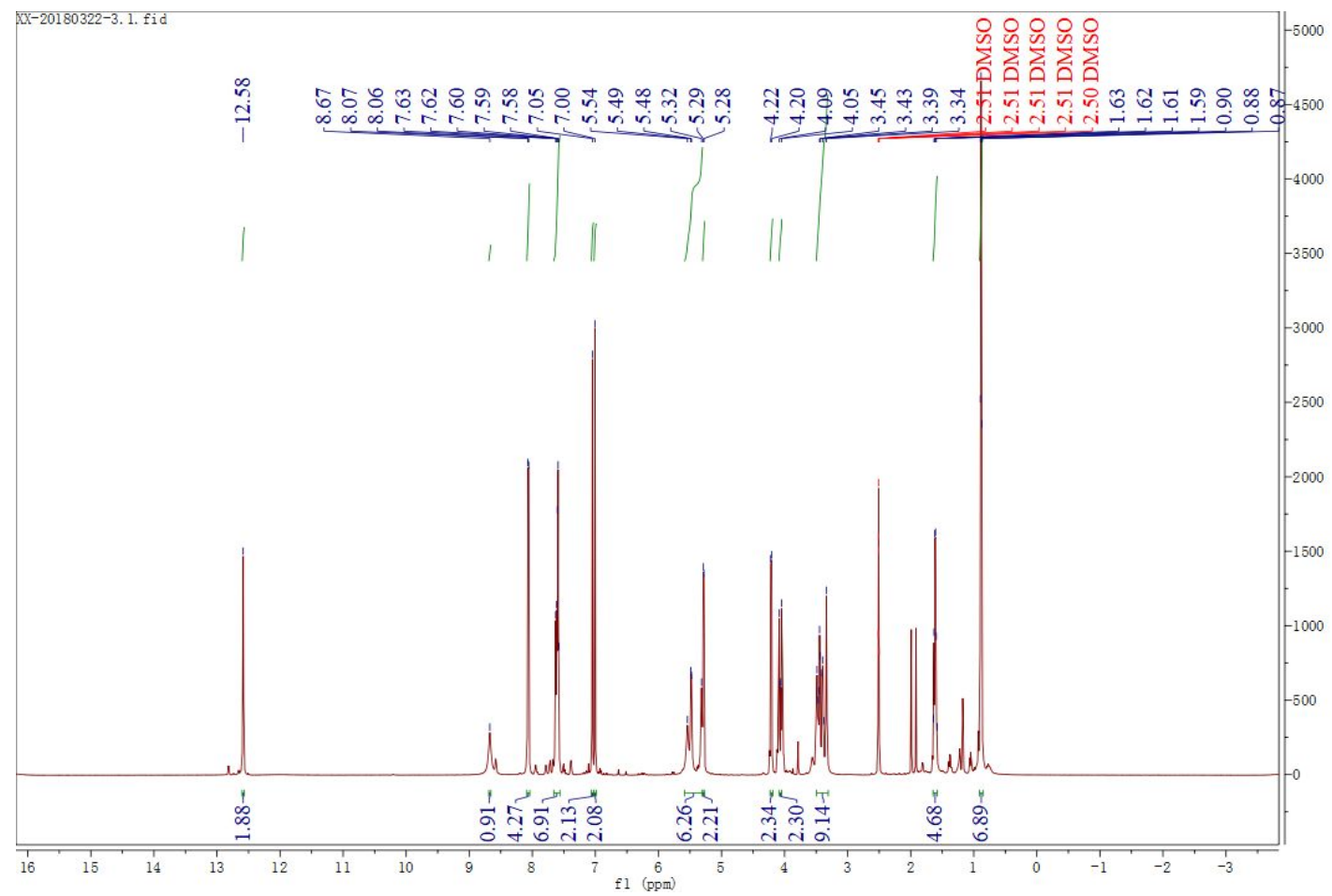

Figure S11. ${ }^{1} \mathrm{H}$ NMR spectrum for compound $\mathbf{2 b}$ in DMSO- $d_{6}(400 \mathrm{MHz})$. 


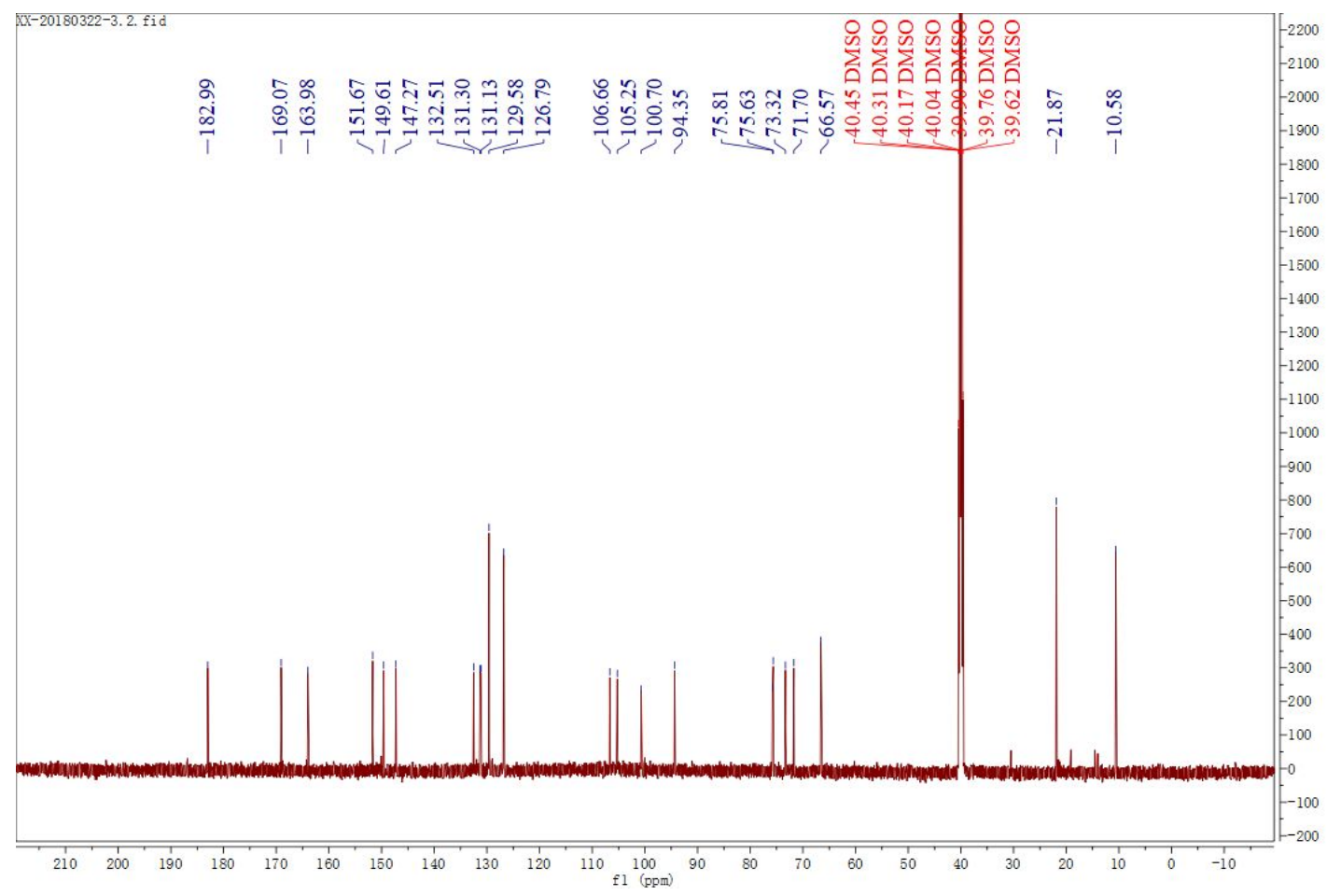

Figure S12. ${ }^{13} \mathrm{C}$ NMR spectrum for compound 2b in DMSO- $d_{6}(100 \mathrm{MHz})$. 

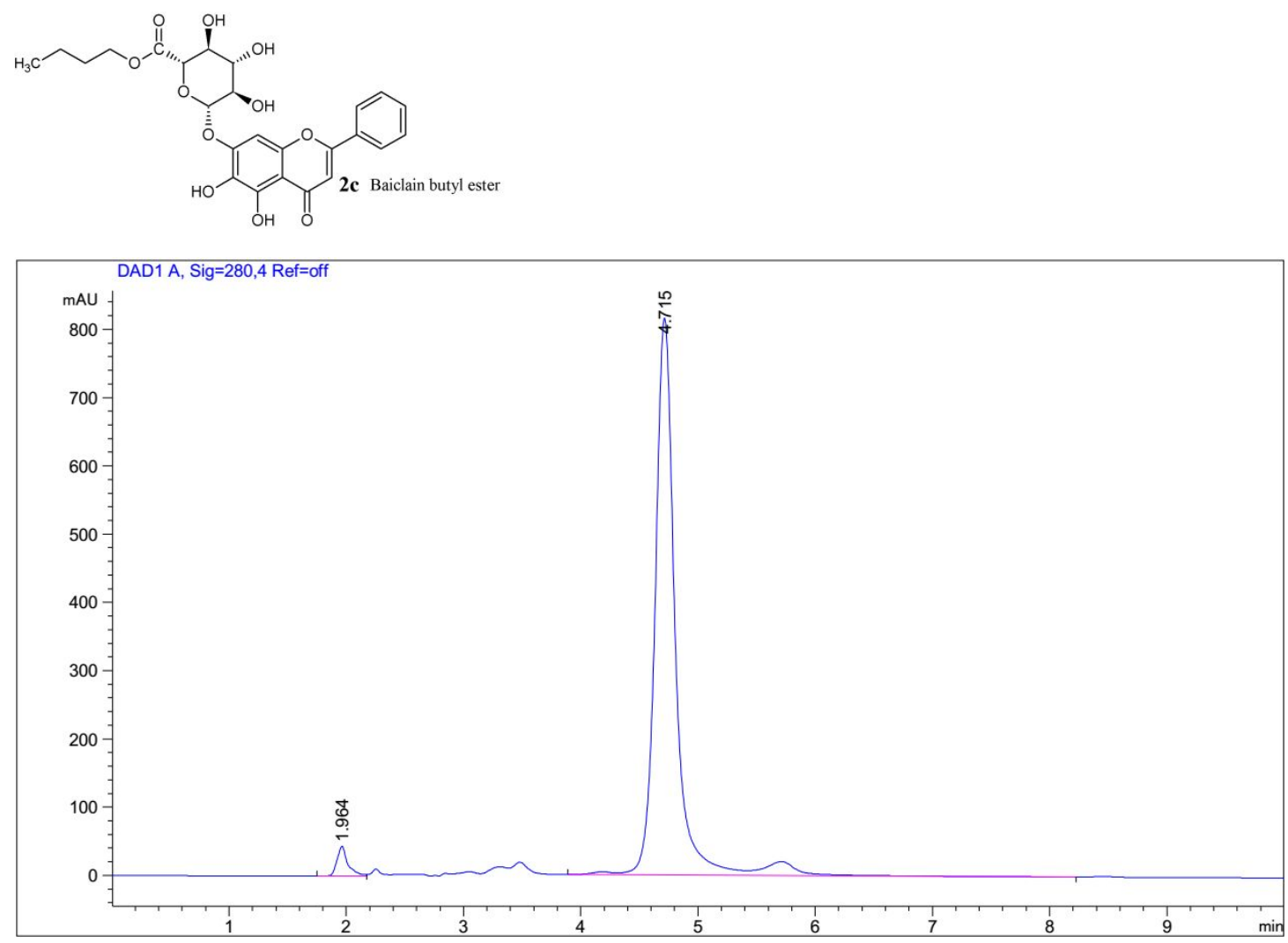

Figure S13. HPLC chromatography result of compound 2c

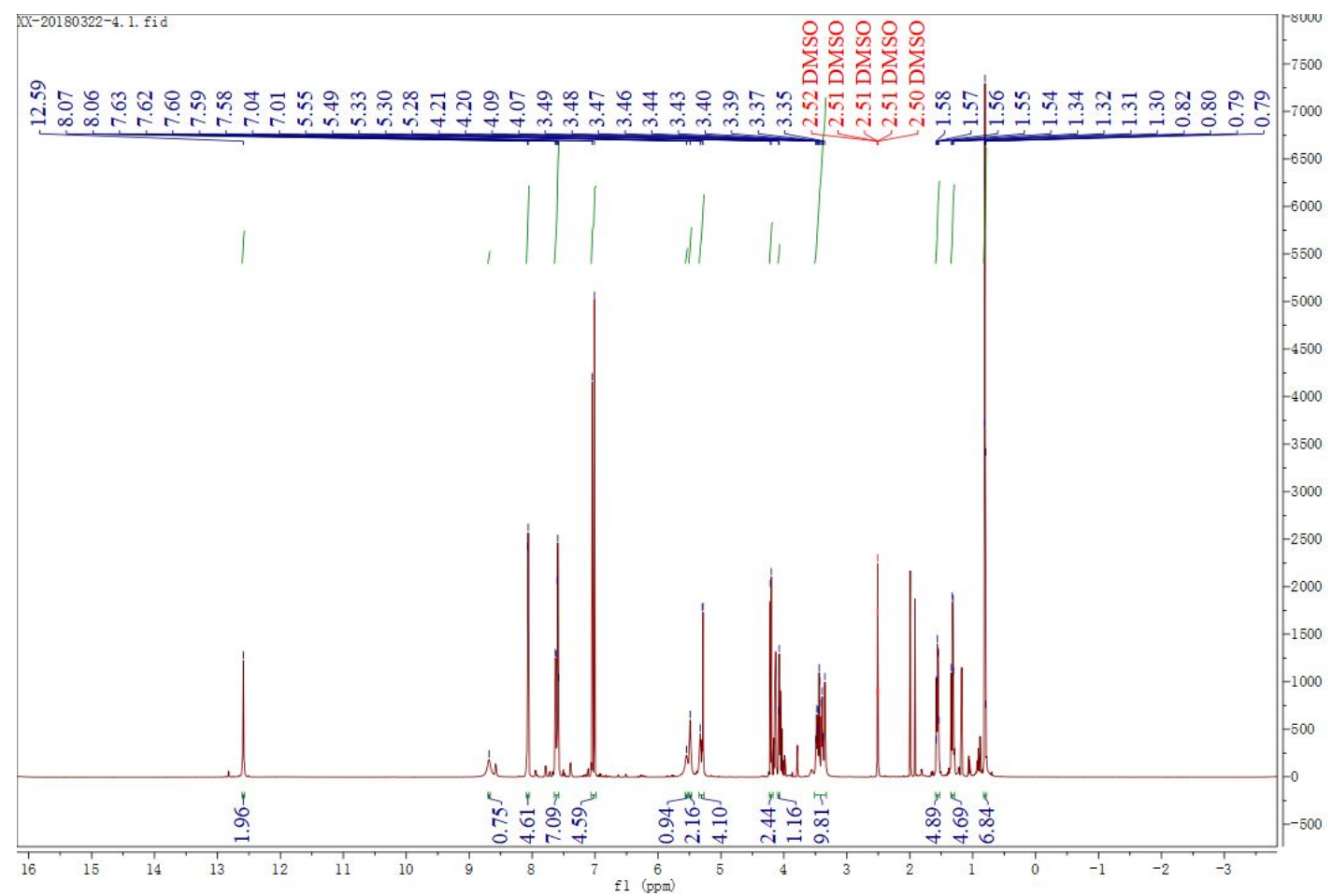

Figure S14. ${ }^{1} \mathrm{H}$ NMR spectrum for compound $2 \mathrm{c}$ in DMSO- $d_{6}(400 \mathrm{MHz})$. 


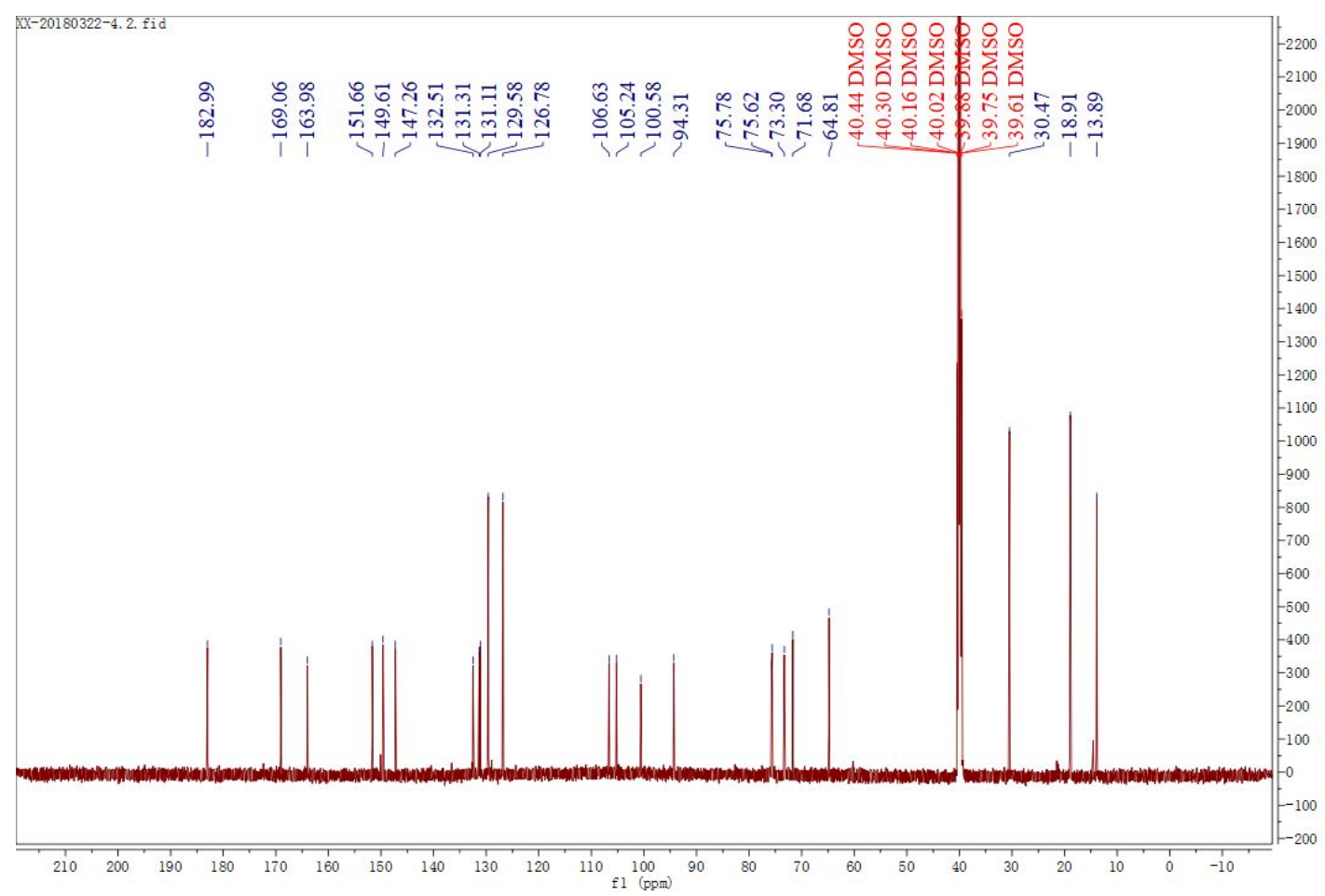

Figure S15. ${ }^{13} \mathrm{C}$ NMR spectrum for compound $2 \mathbf{c}$ in DMSO- $d_{6}(100 \mathrm{MHz})$. 

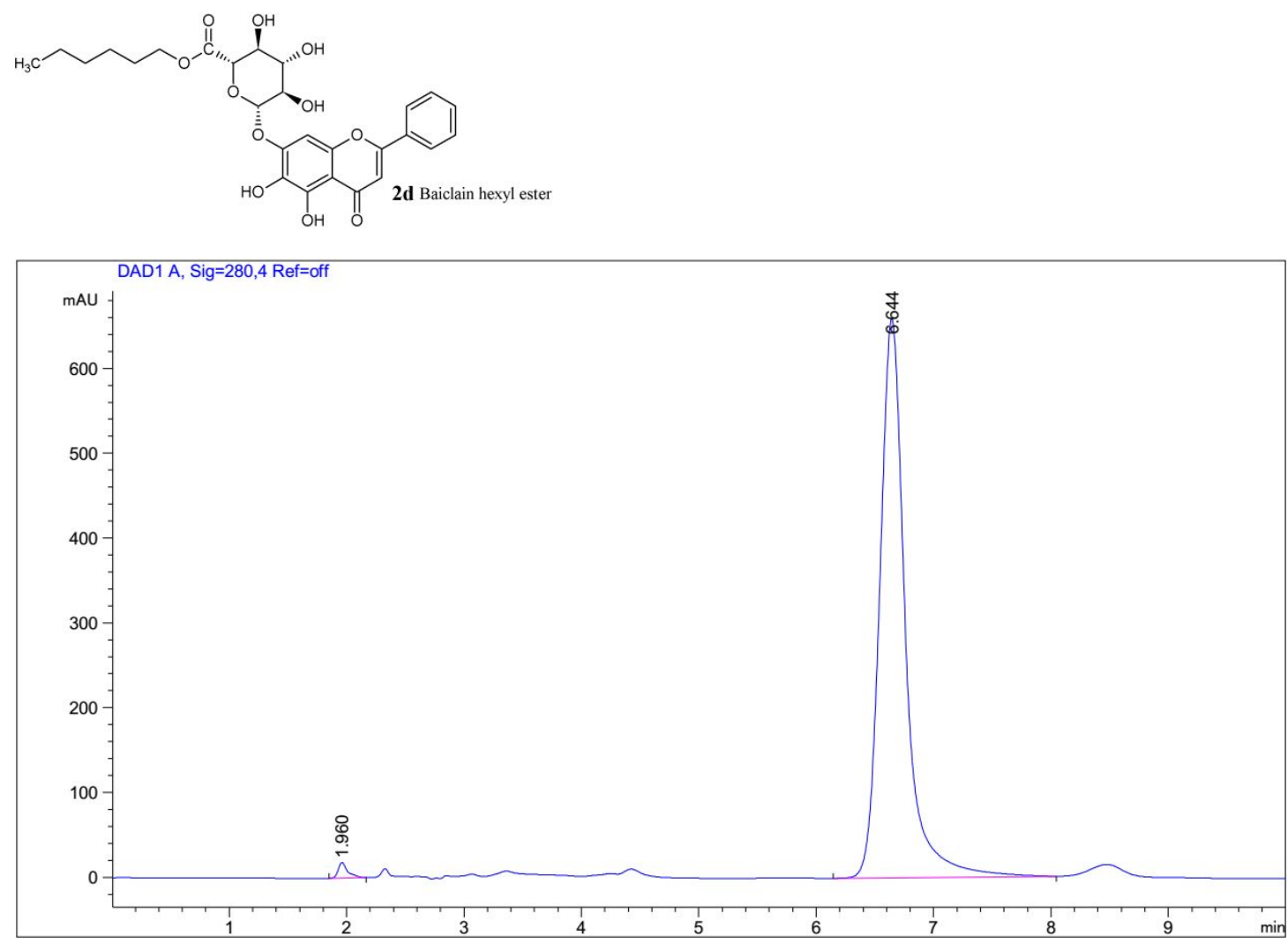

Figure S16. HPLC chromatography result of compound 2d

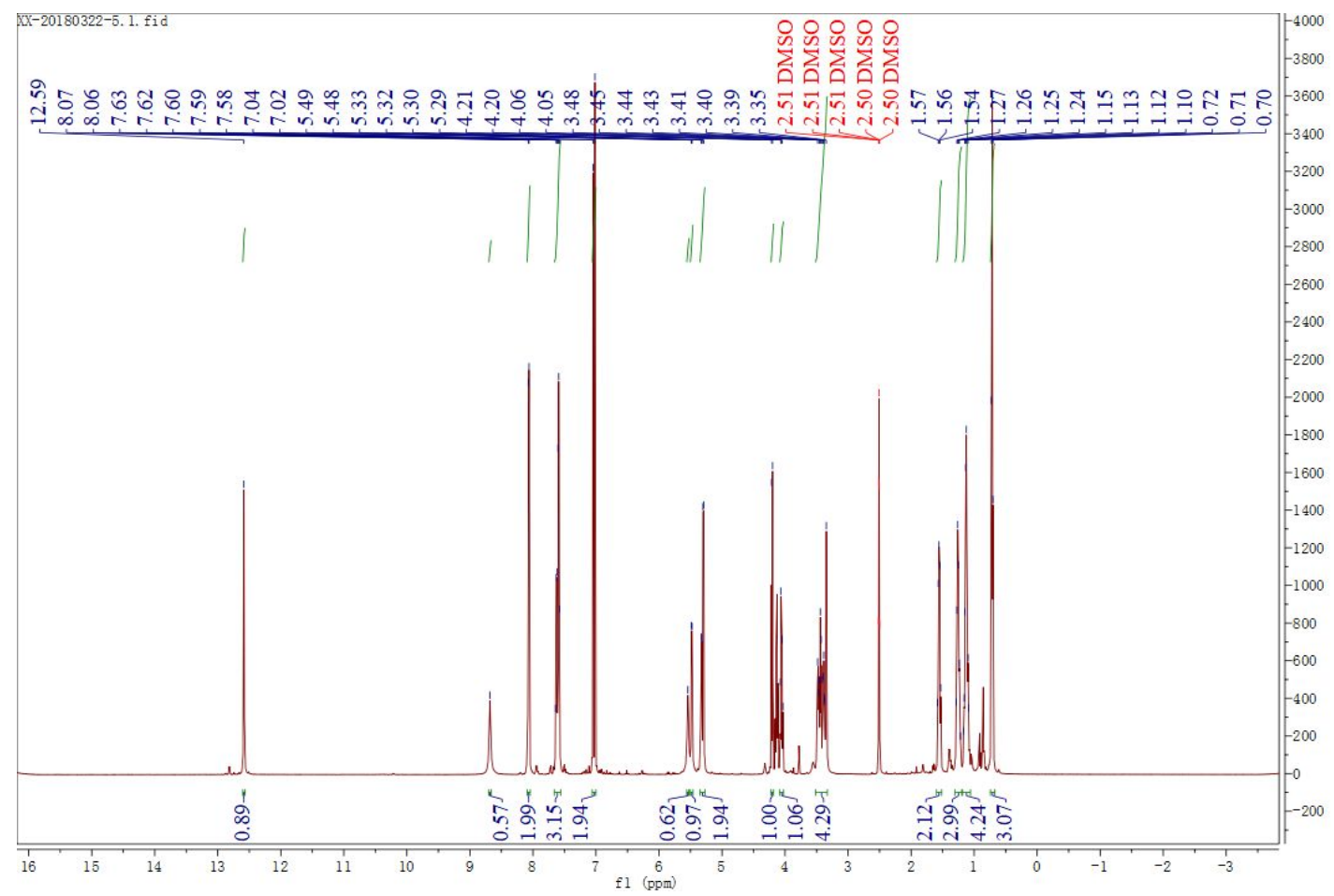

Figure S17. ${ }^{1} \mathrm{H}$ NMR spectrum for compound 2d in DMSO- $d_{6}(400 \mathrm{MHz})$. 


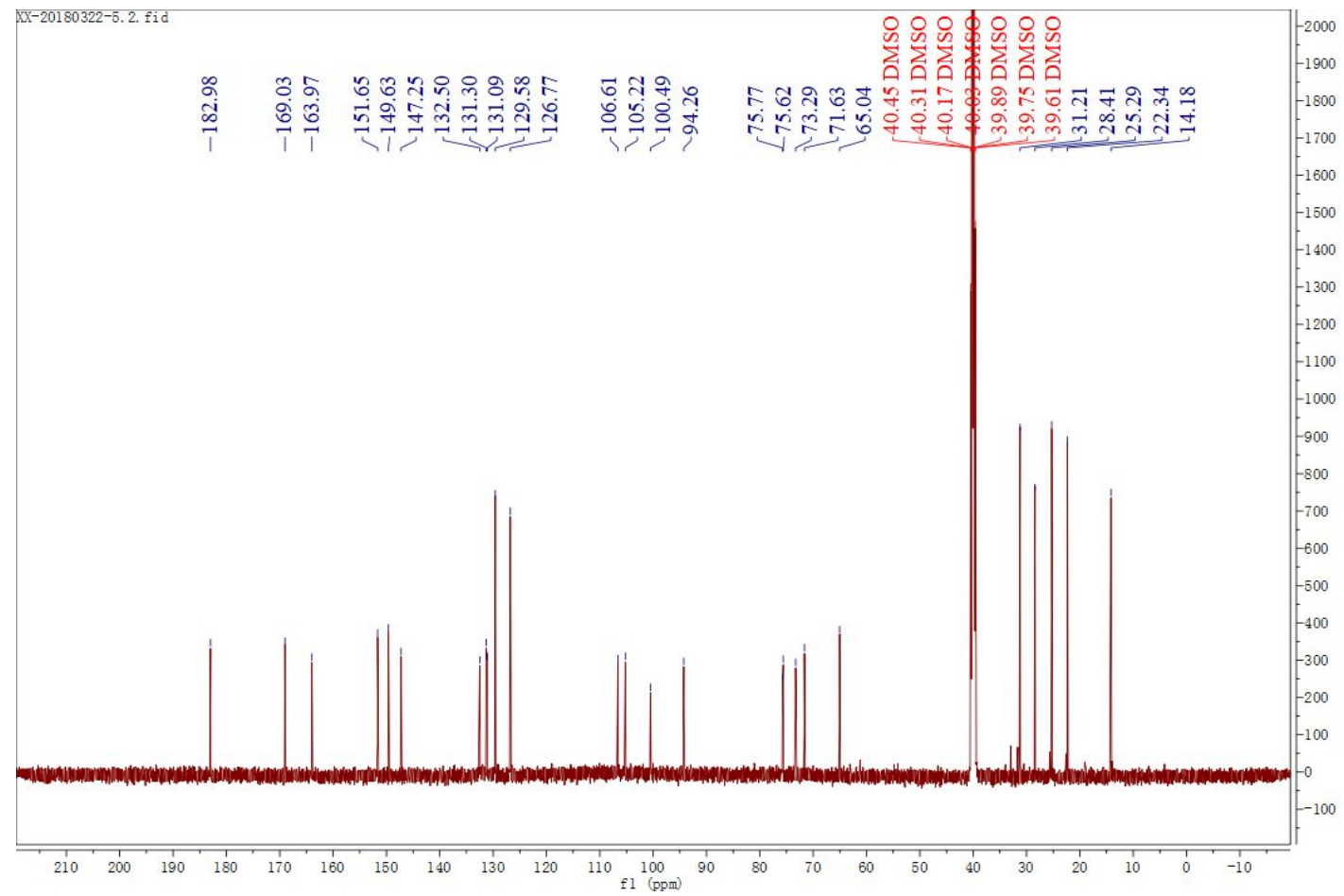

Figure S18. ${ }^{13} \mathrm{C}$ NMR spectrum for compound 2d in DMSO- $d_{6}(100 \mathrm{MHz})$. 

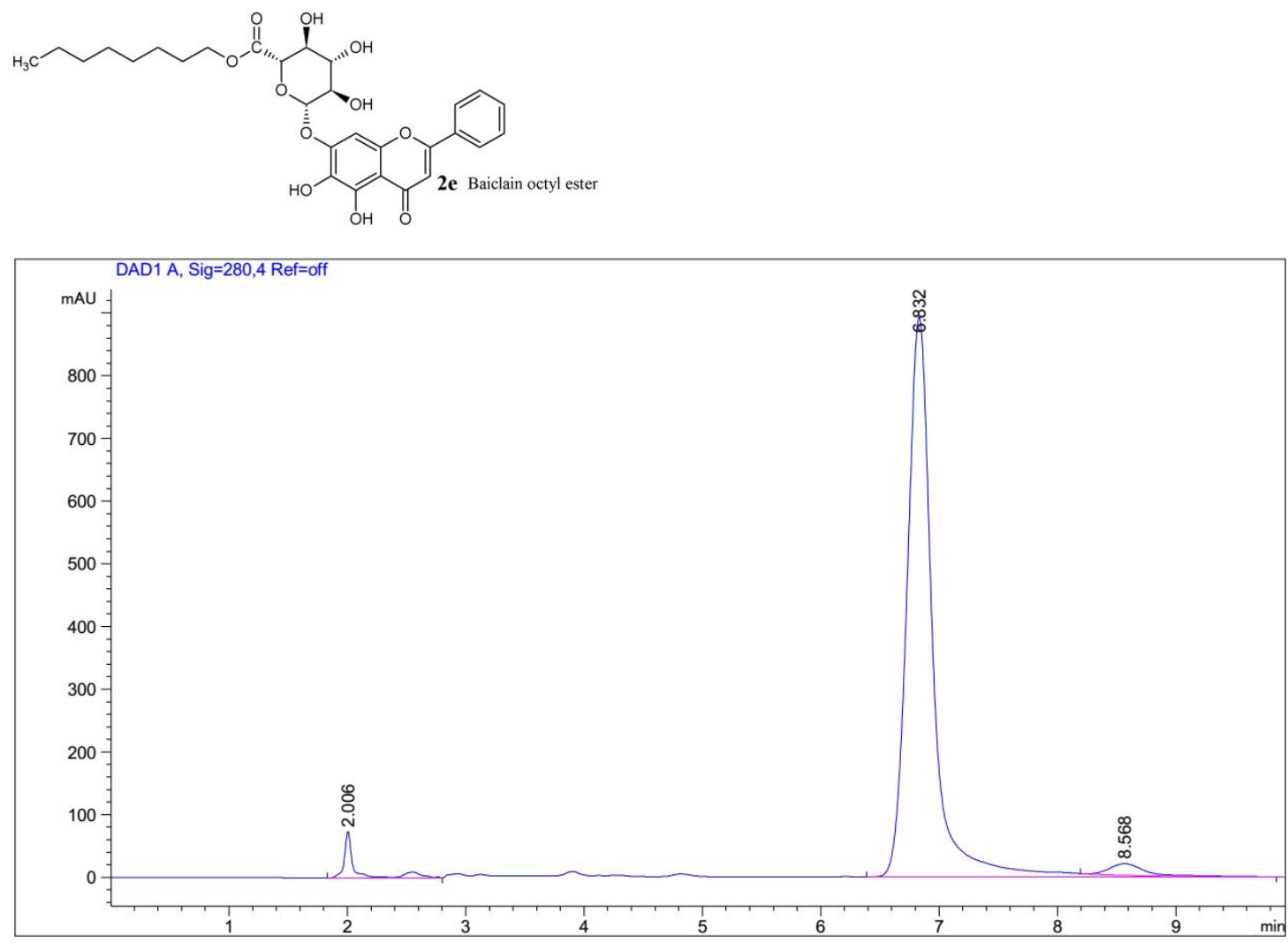

Figure S19. HPLC chromatography result of compound 2e

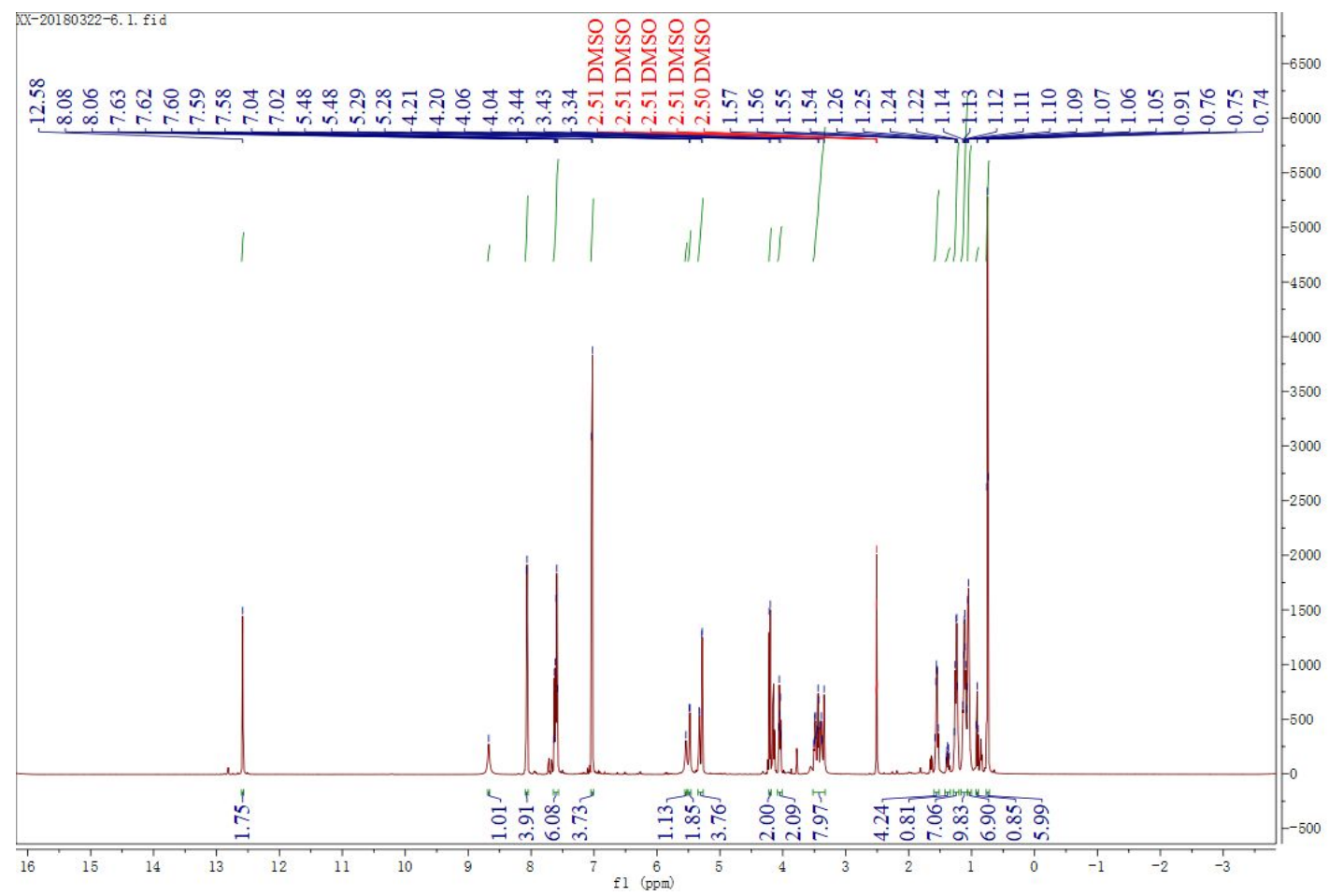

Figure S20. ${ }^{1} \mathrm{H}$ NMR spectrum for compound 2e in DMSO- $d_{6}(400 \mathrm{MHz})$. 


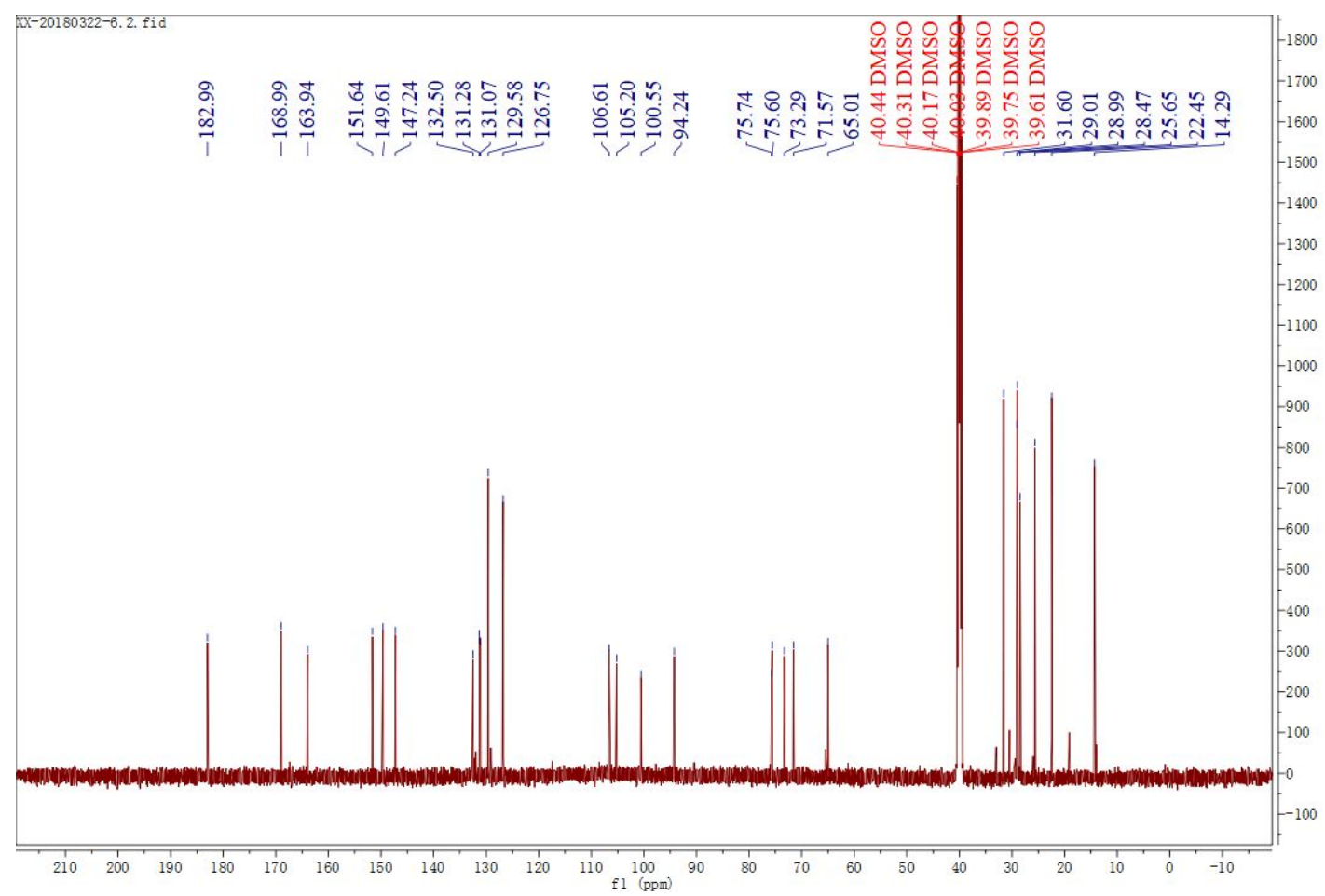

Figure S21. ${ }^{13} \mathrm{C}$ NMR spectrum for compound $2 \mathrm{e}$ in DMSO- $d_{6}(100 \mathrm{MHz})$. 

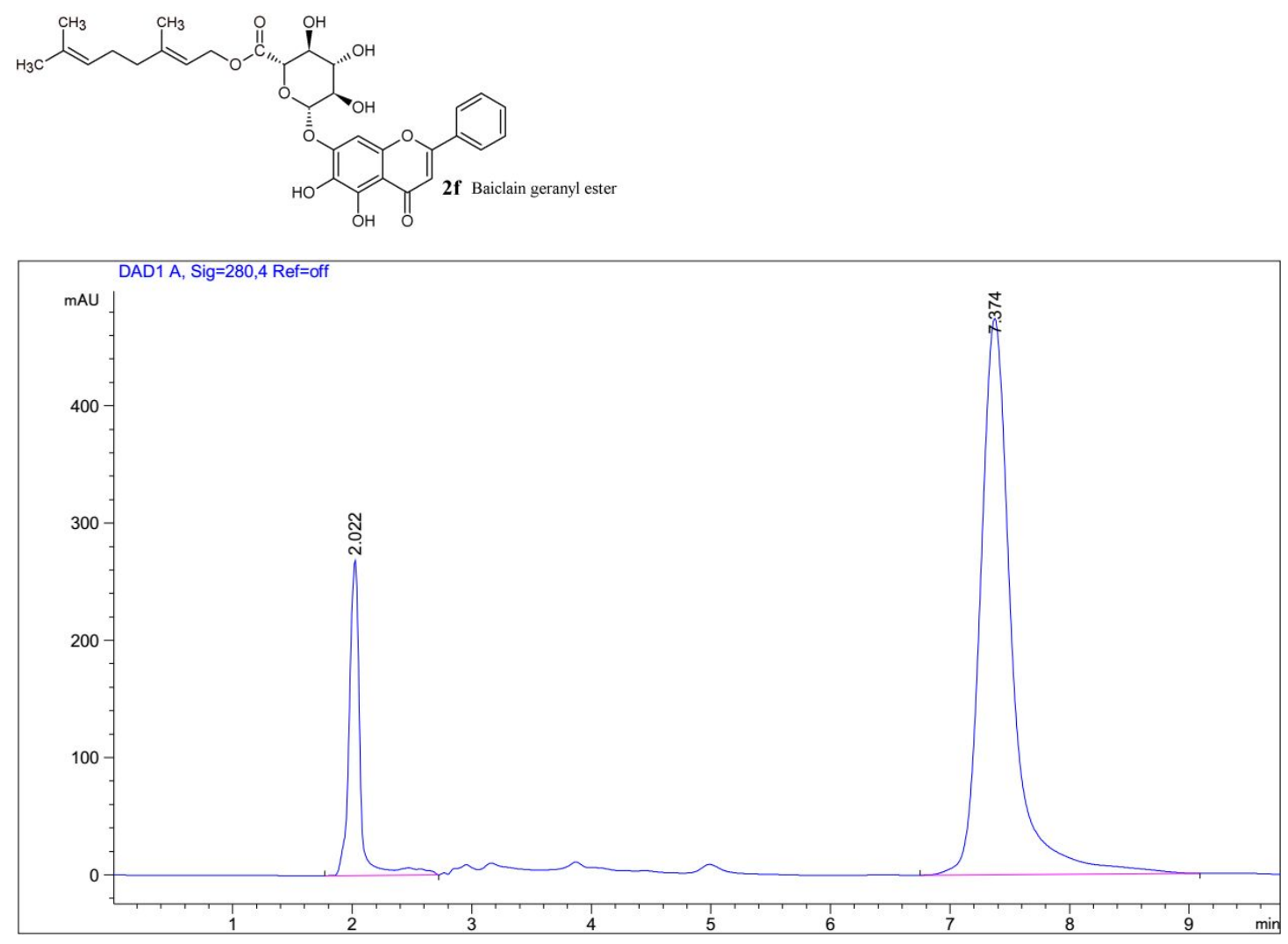

Figure S22. HPLC chromatography result of compound $\mathbf{2 f}$

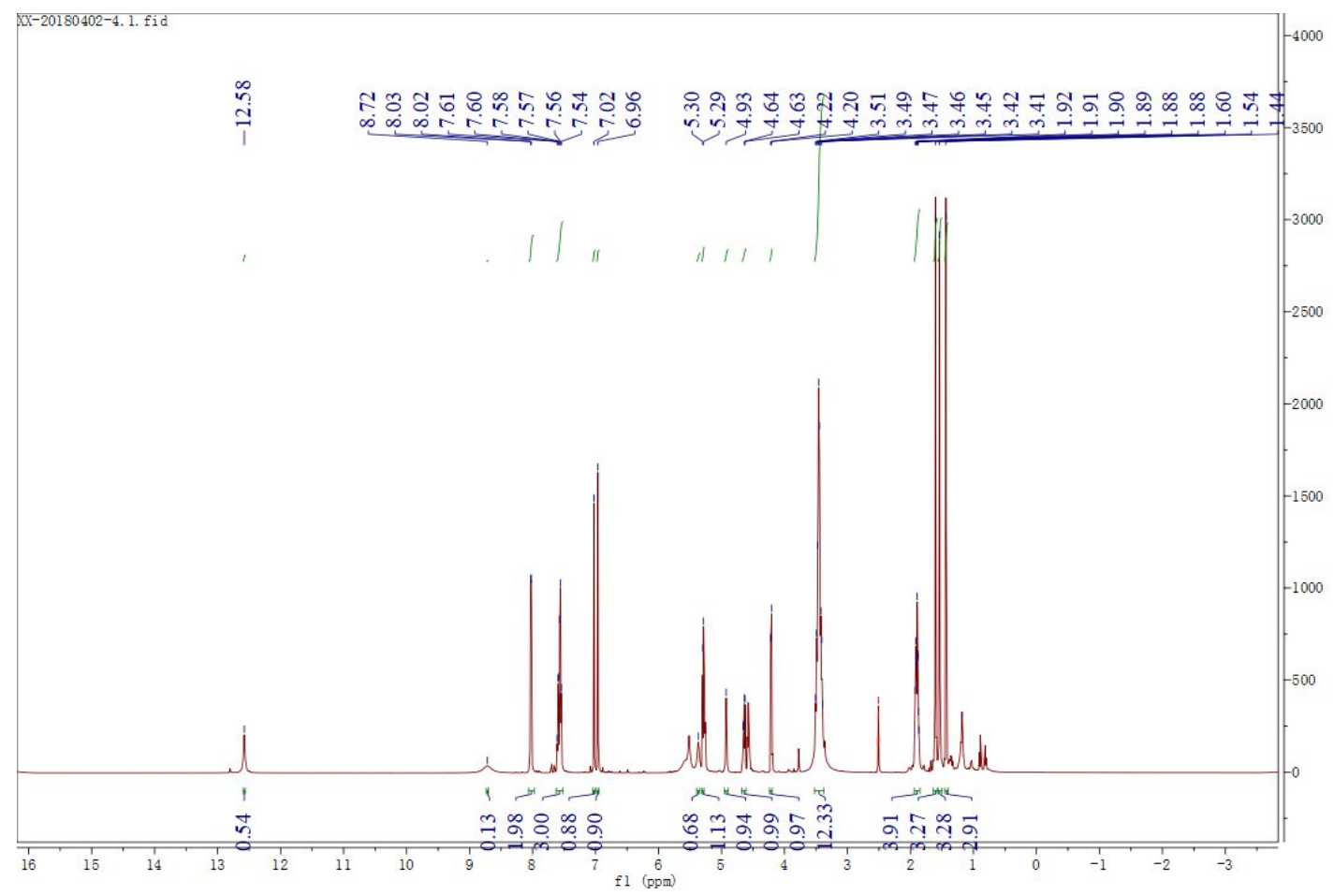

Figure S23. ${ }^{1} \mathrm{H}$ NMR spectrum for compound $2 \mathbf{f}$ in DMSO- $d_{6}(400 \mathrm{MHz})$. 


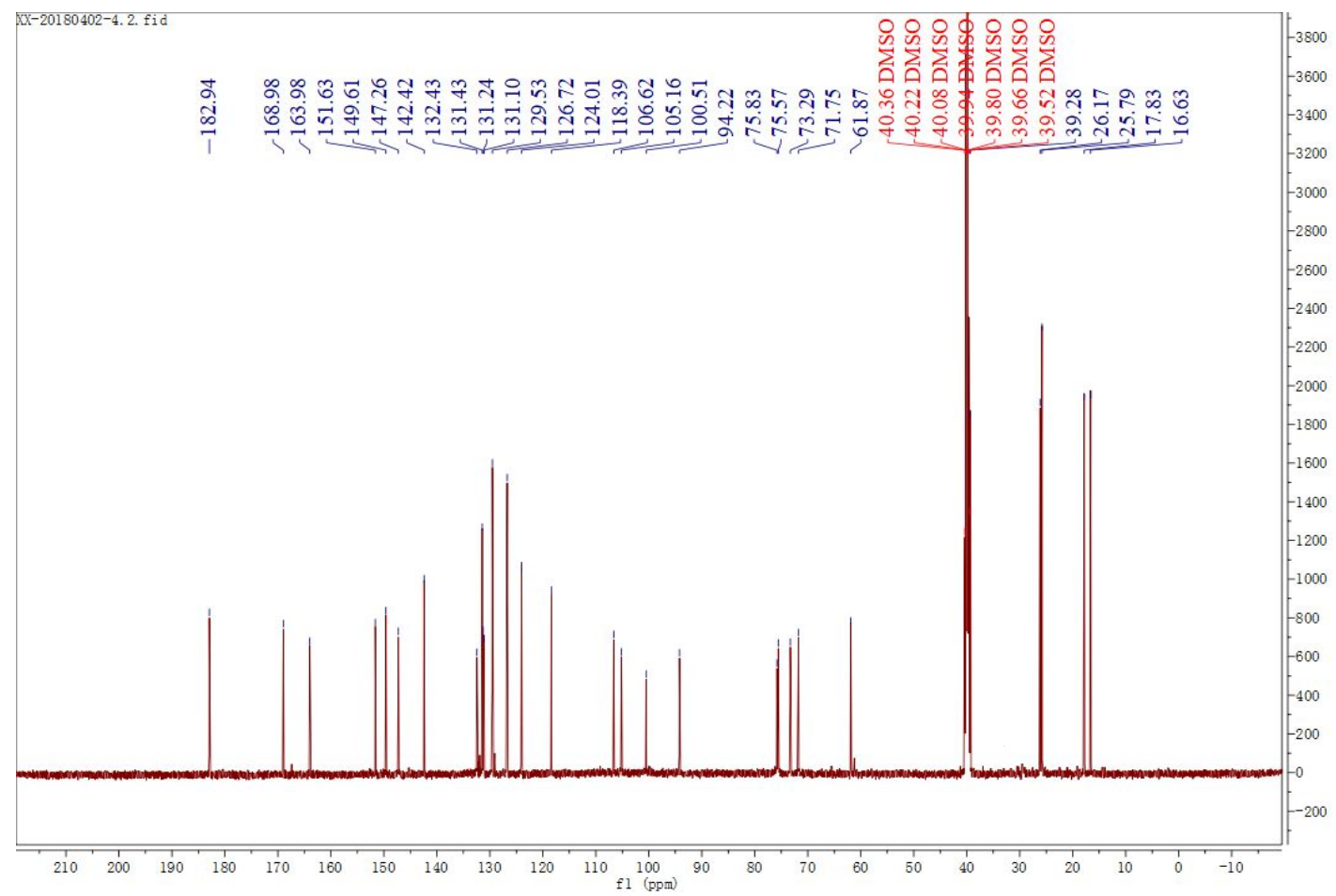

Figure S24. ${ }^{13} \mathrm{C}$ NMR spectrum for compound $2 \mathbf{f}$ in DMSO- $d_{6}(100 \mathrm{MHz})$. 

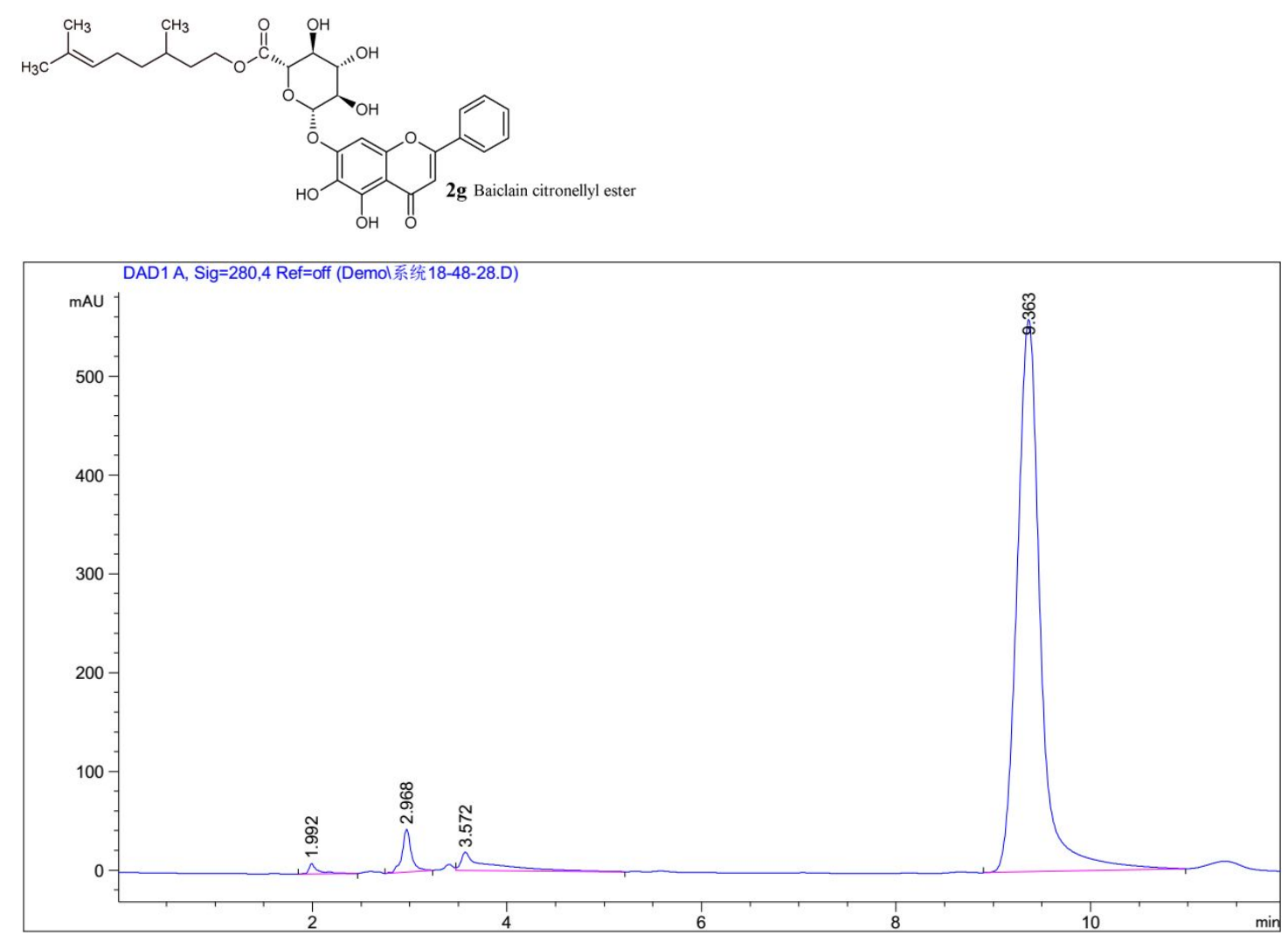

Figure S25. HPLC chromatography result of compound $\mathbf{2 g}$

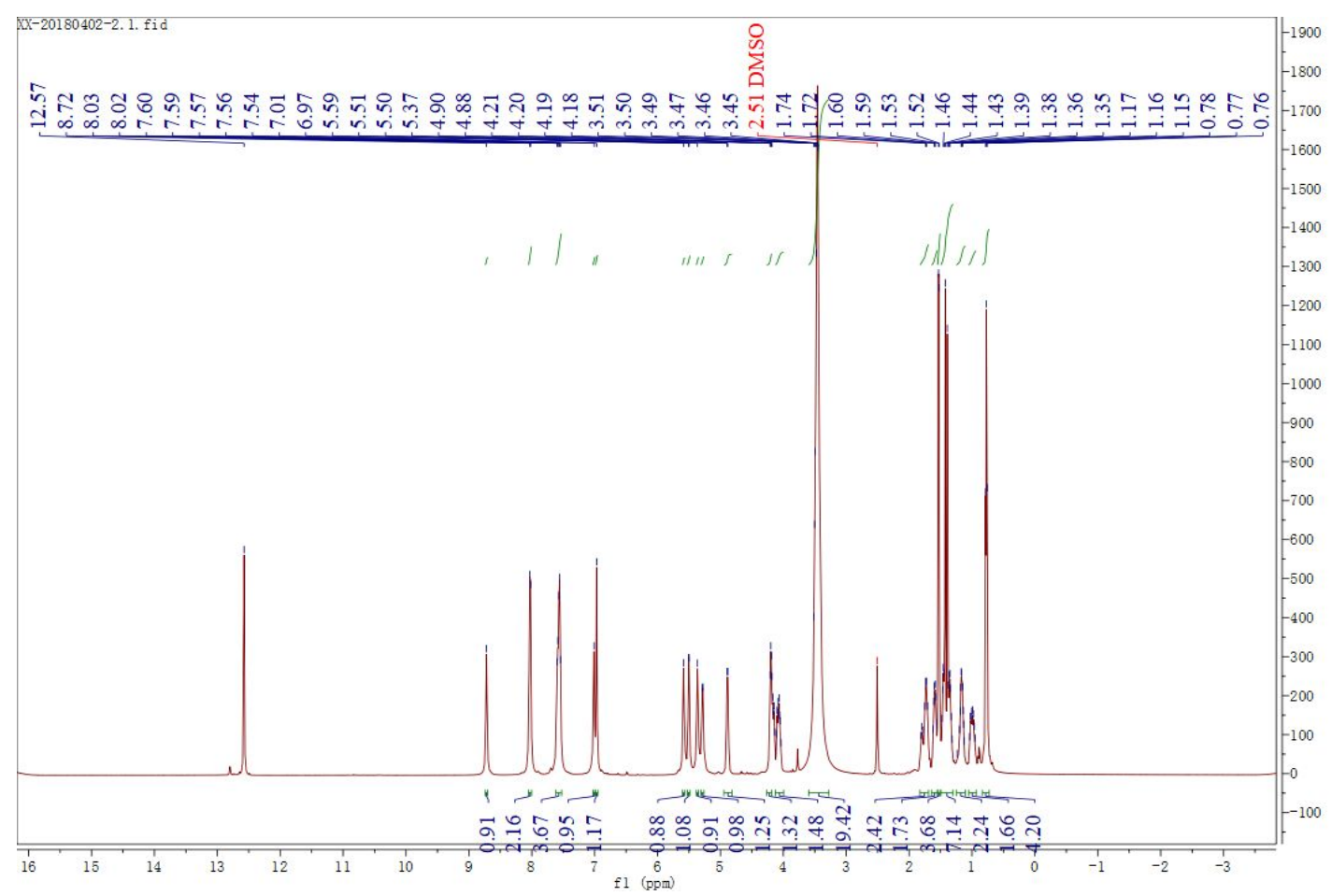

Figure S26. ${ }^{1} \mathrm{H}$ NMR spectrum for compound $2 \mathrm{~g}$ in DMSO- $d_{6}(400 \mathrm{MHz})$. 


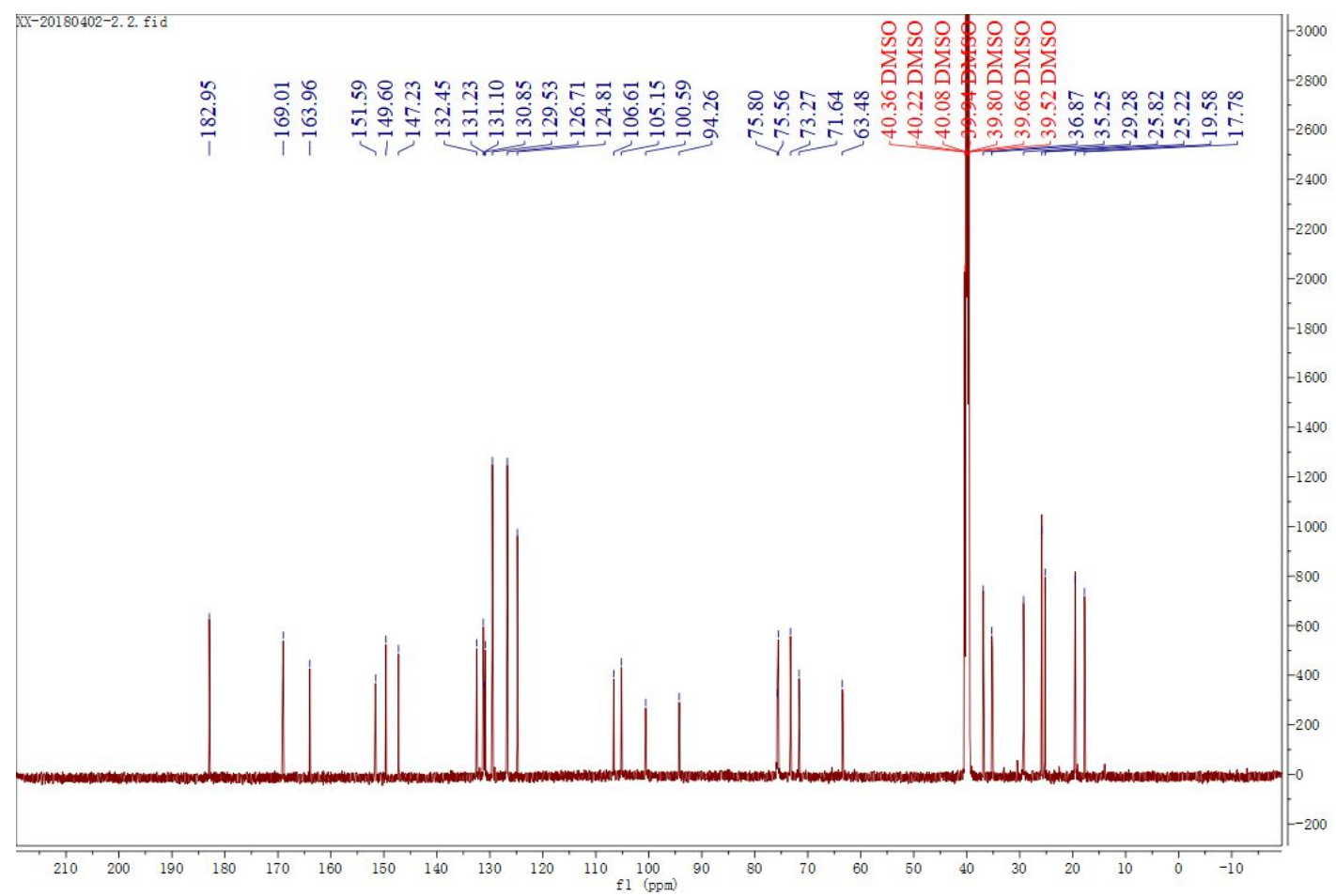

Figure S27. ${ }^{13} \mathrm{C}$ NMR spectrum for compound $2 \mathrm{~g}$ in DMSO- $d_{6}(100 \mathrm{MHz})$. 

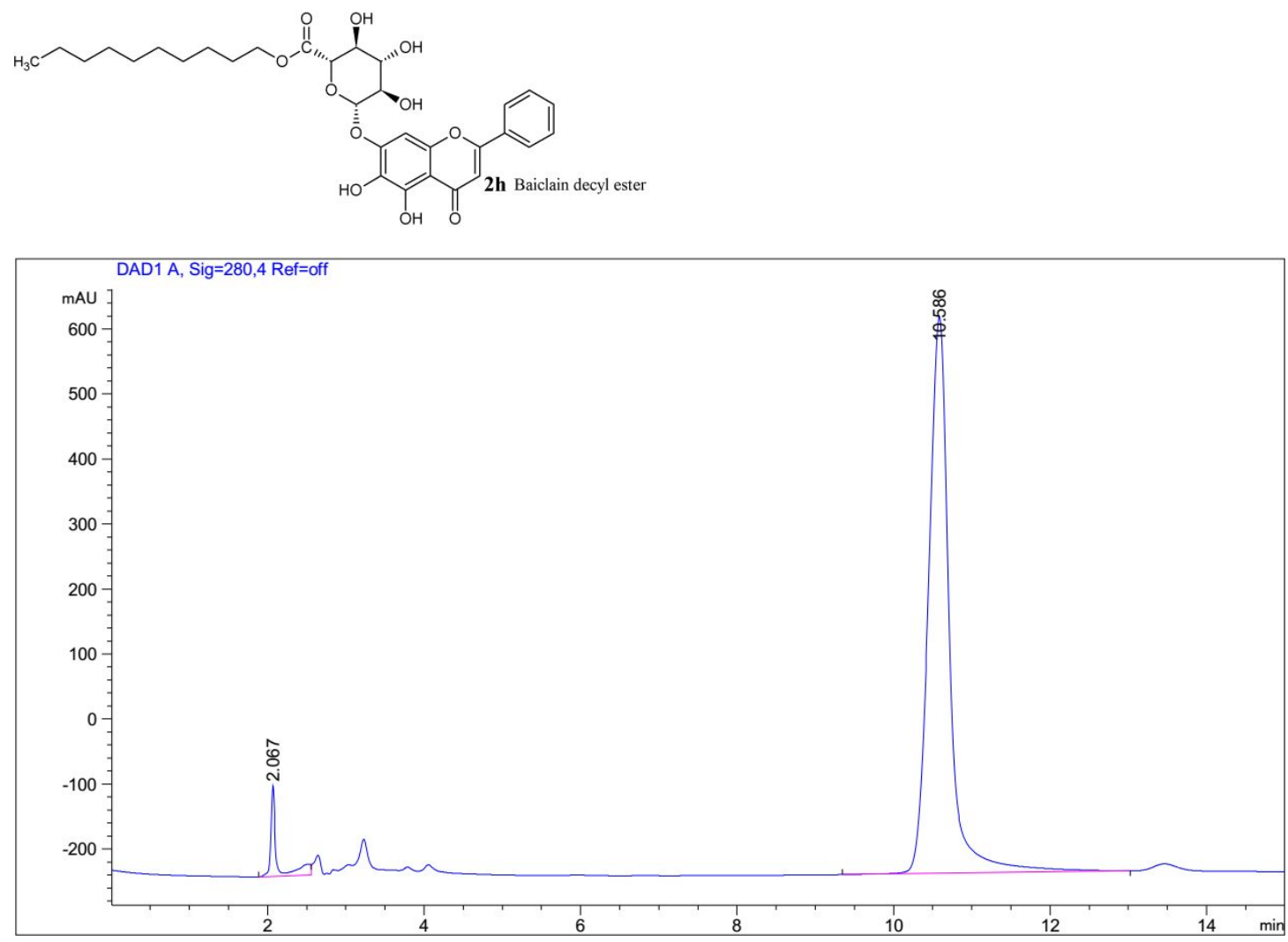

Figure S28. HPLC chromatography result of compound $\mathbf{2 h}$

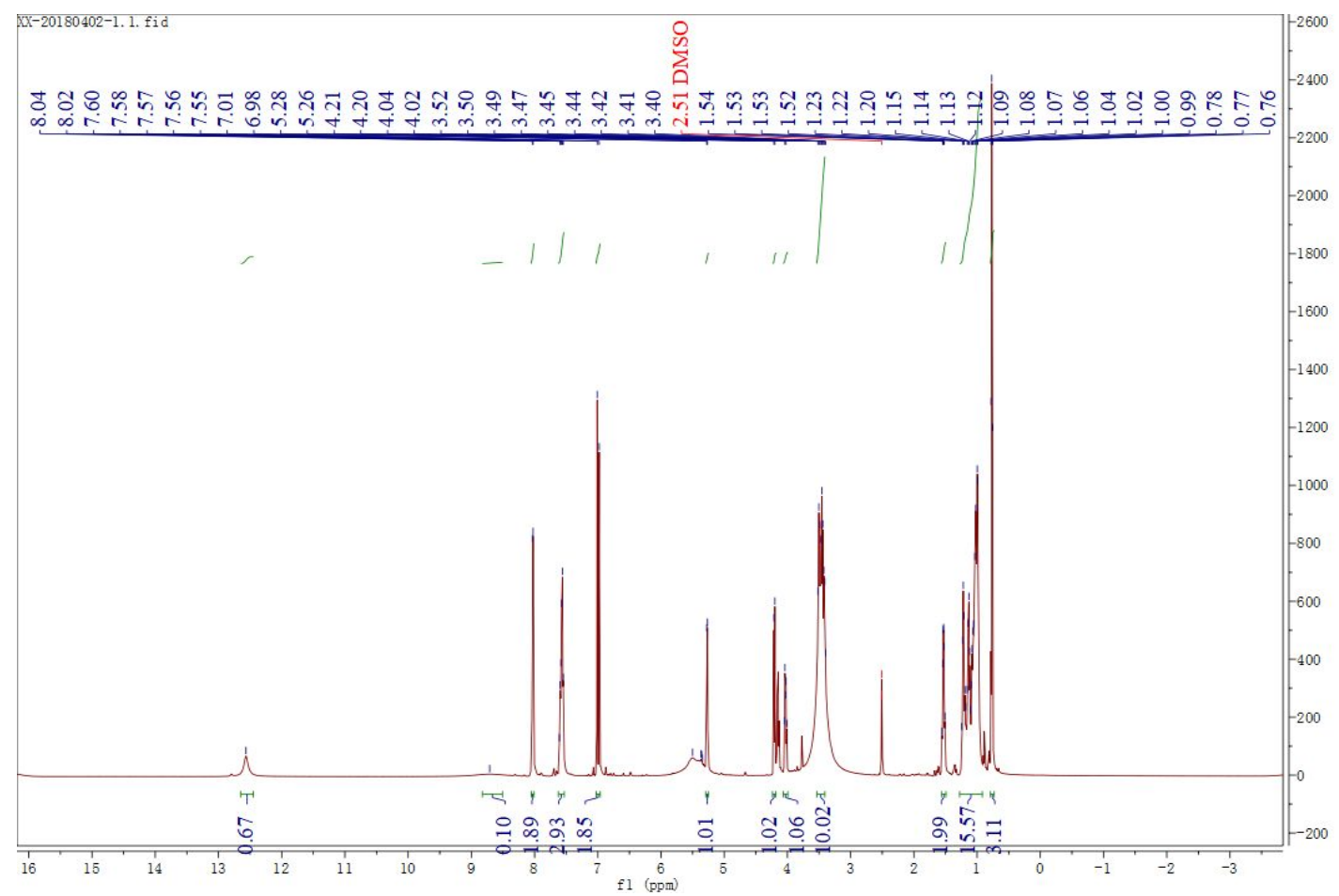

Figure S29. ${ }^{1} \mathrm{H}$ NMR spectrum for compound $\mathbf{2 h}$ in DMSO- $d_{6}(400 \mathrm{MHz})$. 


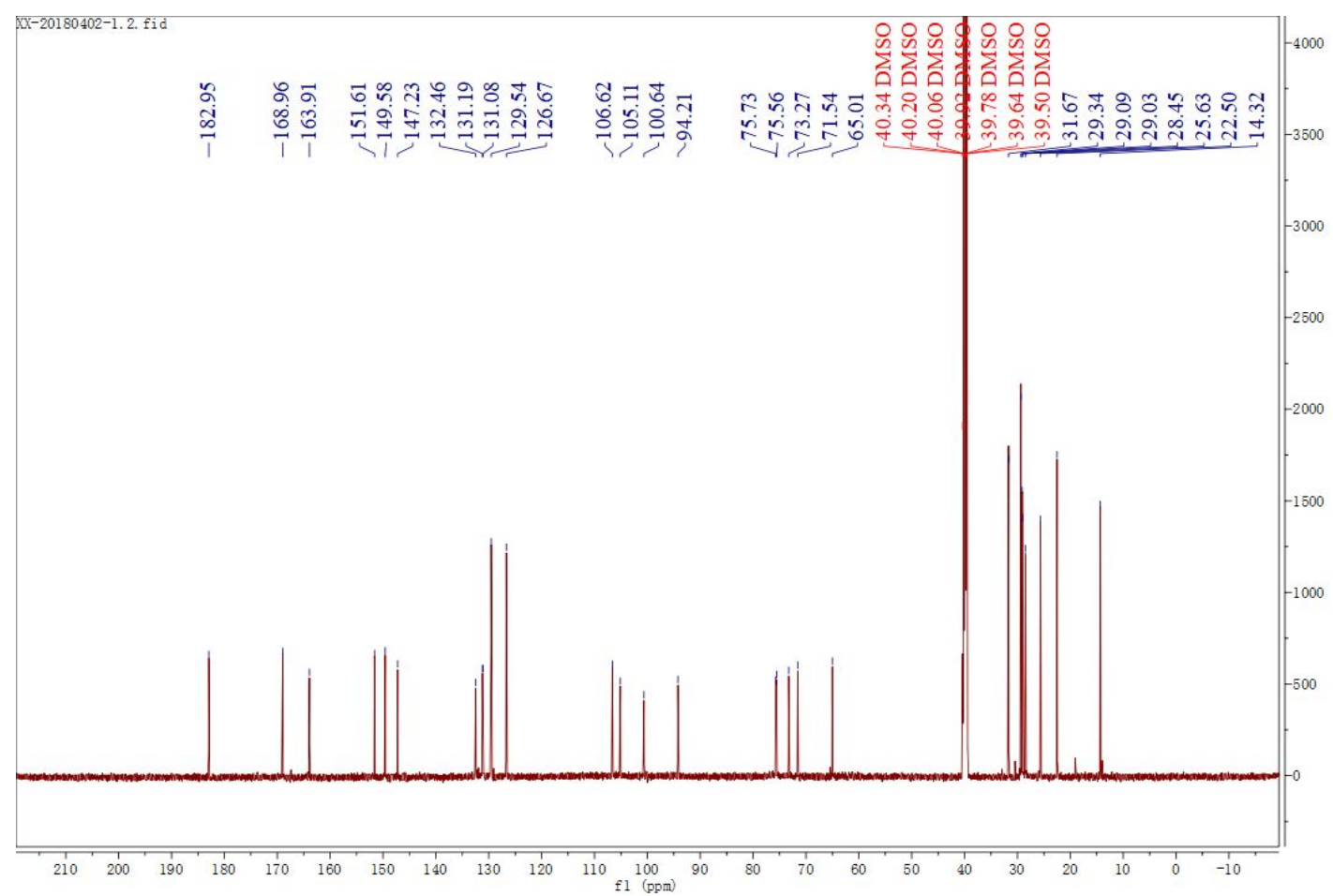

Figure S30. ${ }^{13} \mathrm{C}$ NMR spectrum for compound $\mathbf{2 h}$ in DMSO- $d_{6}(100 \mathrm{MHz})$. 

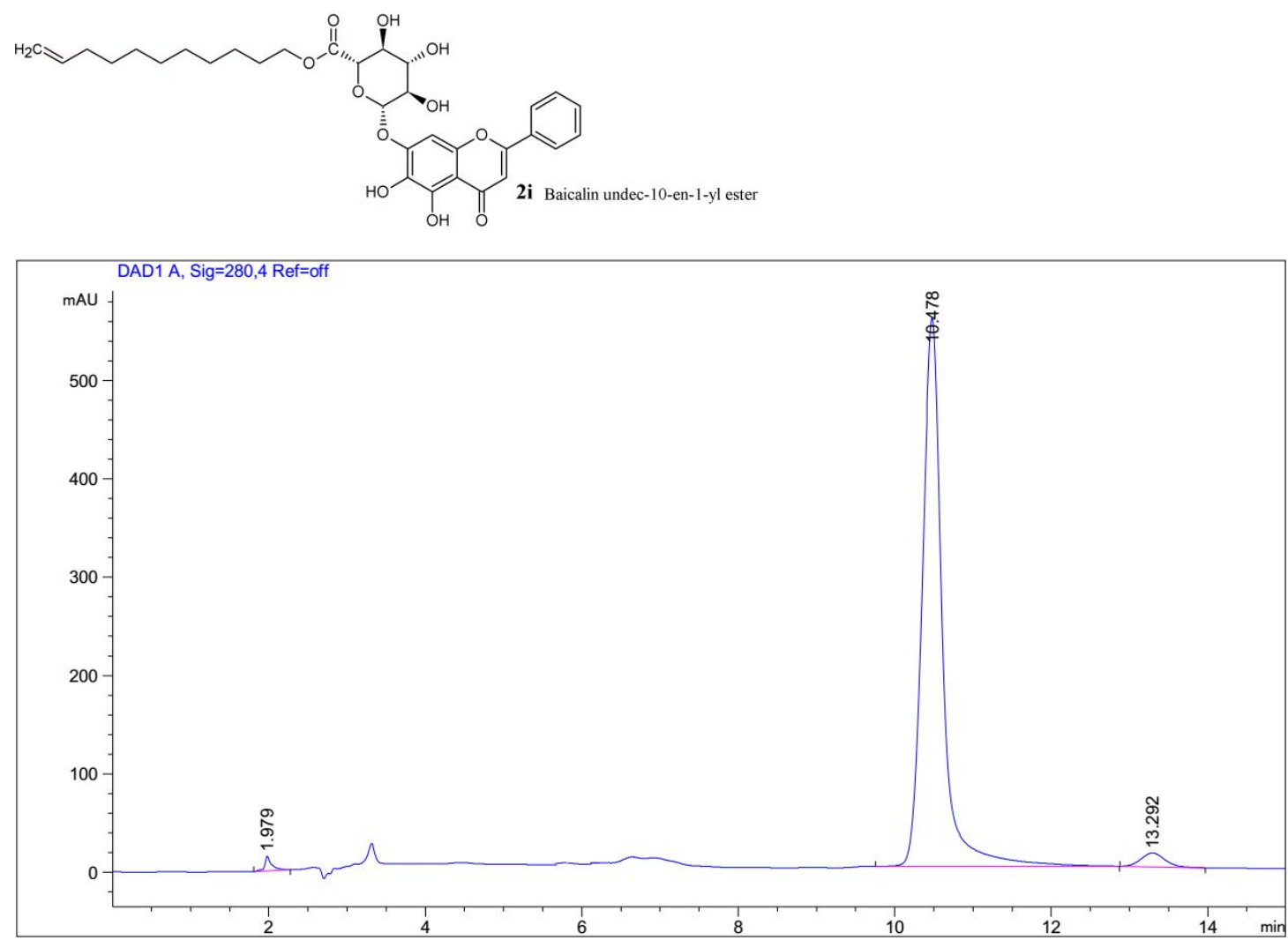

Figure S31. HPLC chromatography result of compound 2i

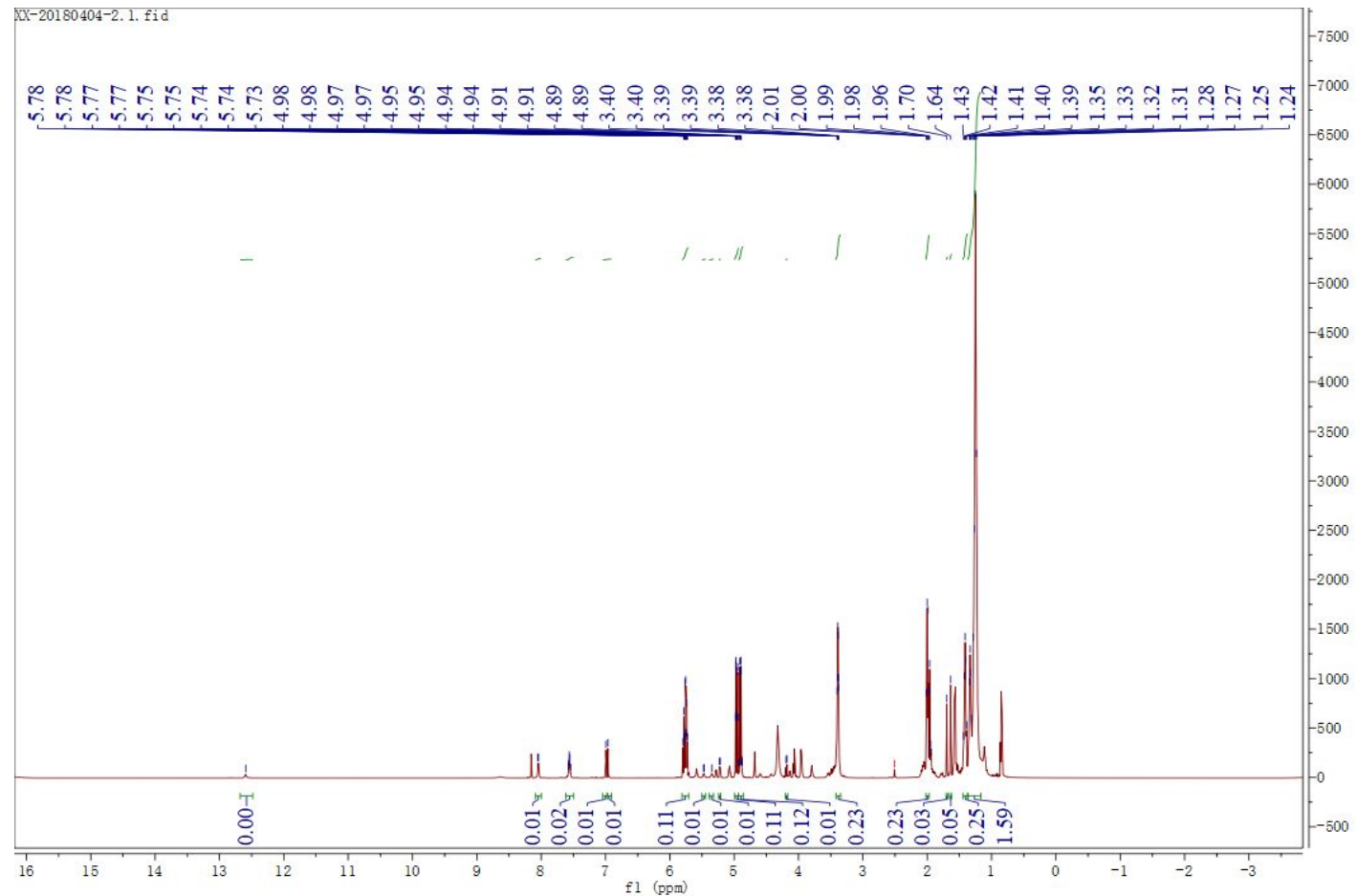

Figure S32. ${ }^{1} \mathrm{H}$ NMR spectrum for compound $2 \mathbf{i}$ in DMSO- $d_{6}(400 \mathrm{MHz})$. 


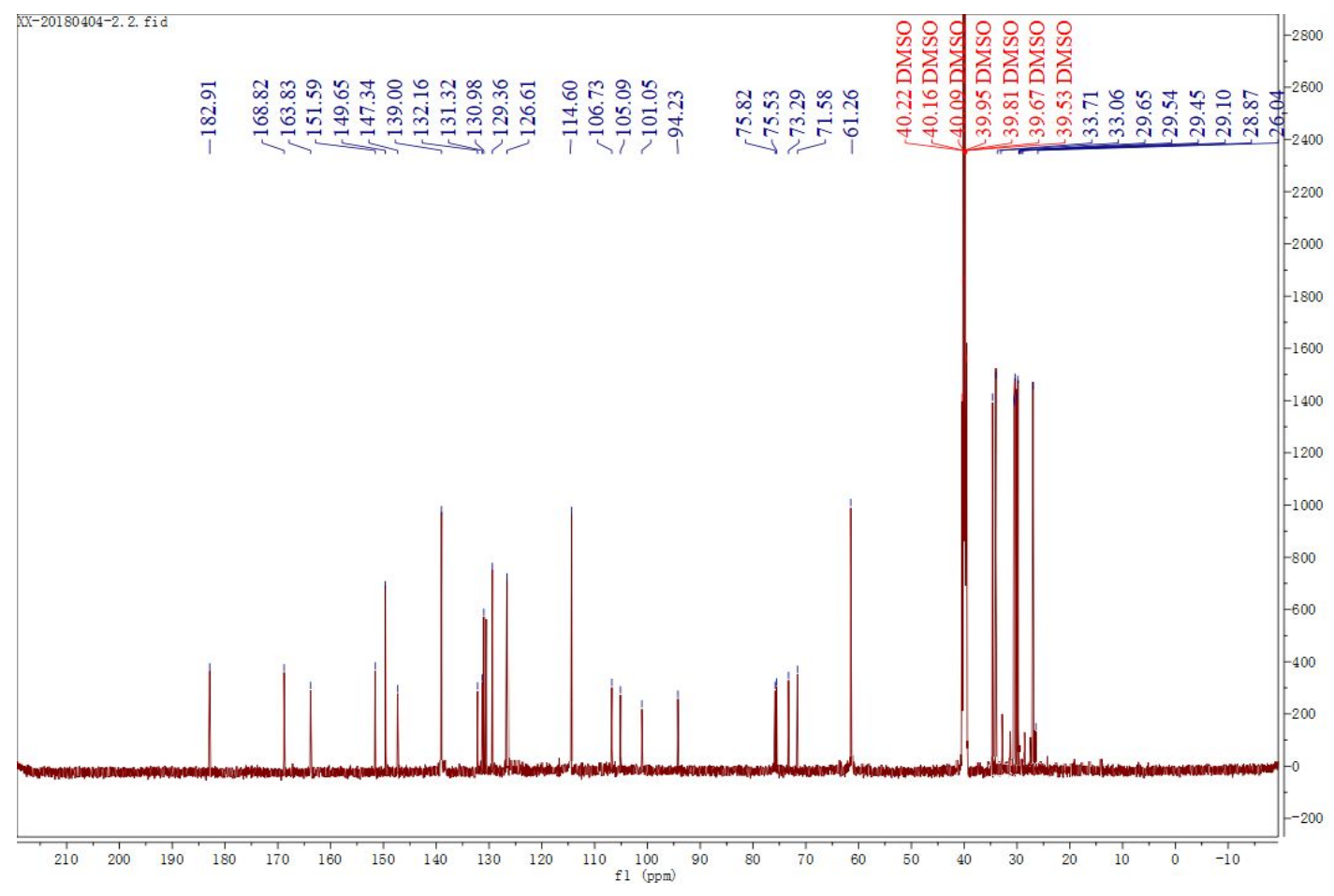

Figure S33. ${ }^{13} \mathrm{C}$ NMR spectrum for compound $2 \mathbf{i}$ in DMSO- $d_{6}(100 \mathrm{MHz})$. 

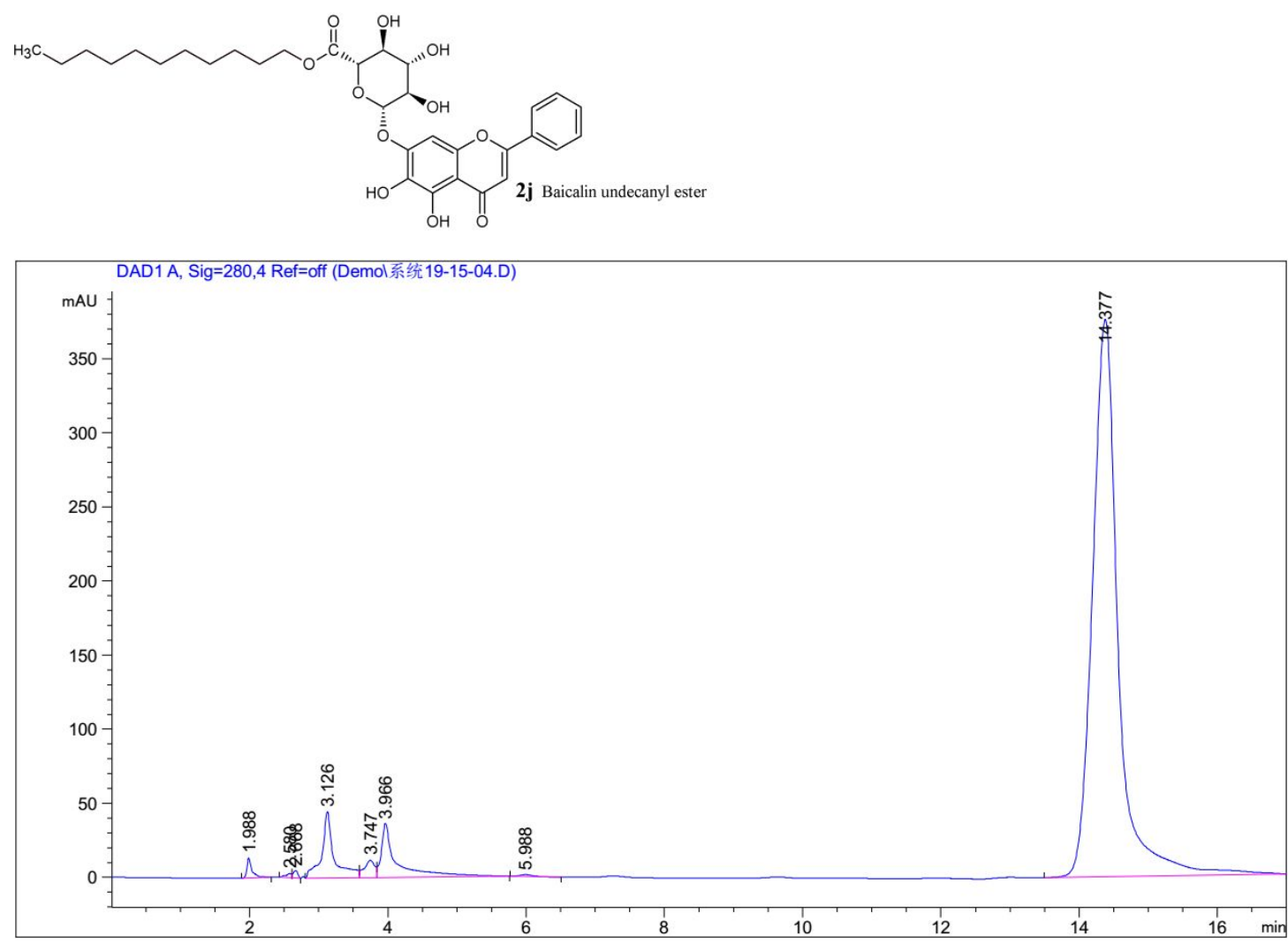

Figure S34. HPLC chromatography result of compound $\mathbf{2 j}$

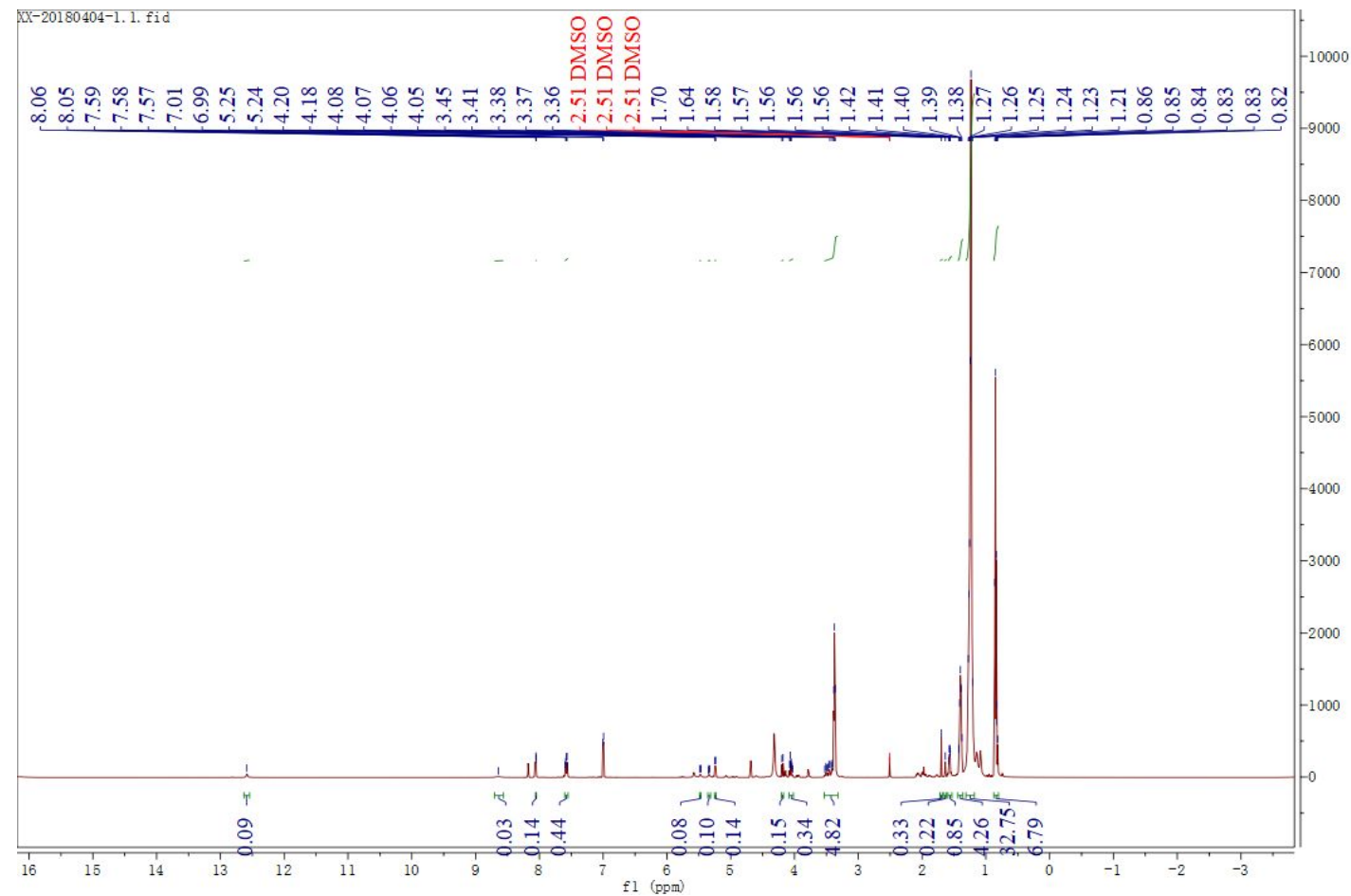

Figure S35. ${ }^{1} \mathrm{H}$ NMR spectrum for compound $\mathbf{2 j}$ in DMSO- $d_{6}(400 \mathrm{MHz})$. 


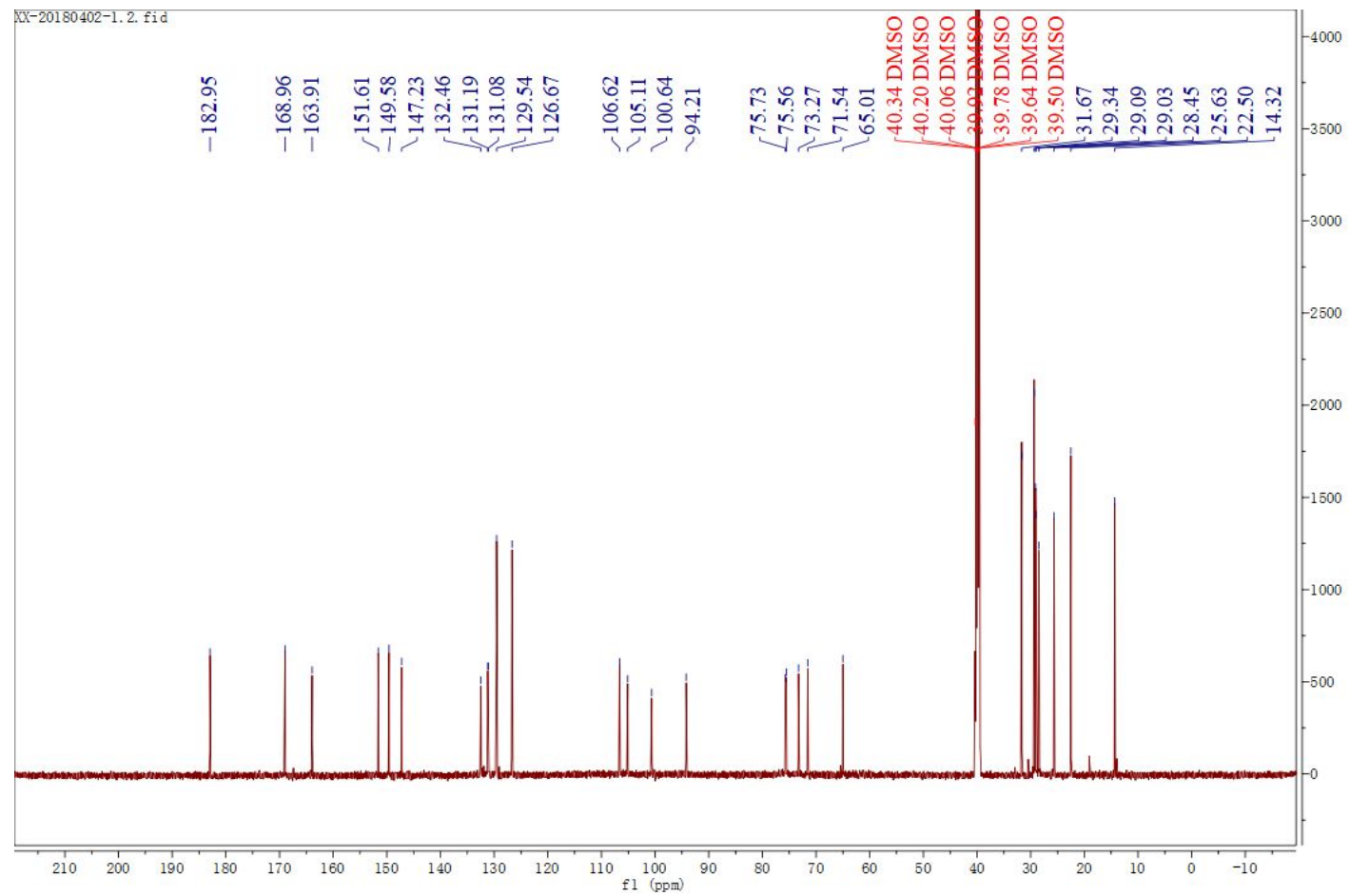

Figure S36. ${ }^{13} \mathrm{C}$ NMR spectrum for compound $\mathbf{2 j}$ in DMSO- $d_{6}(100 \mathrm{MHz})$. 

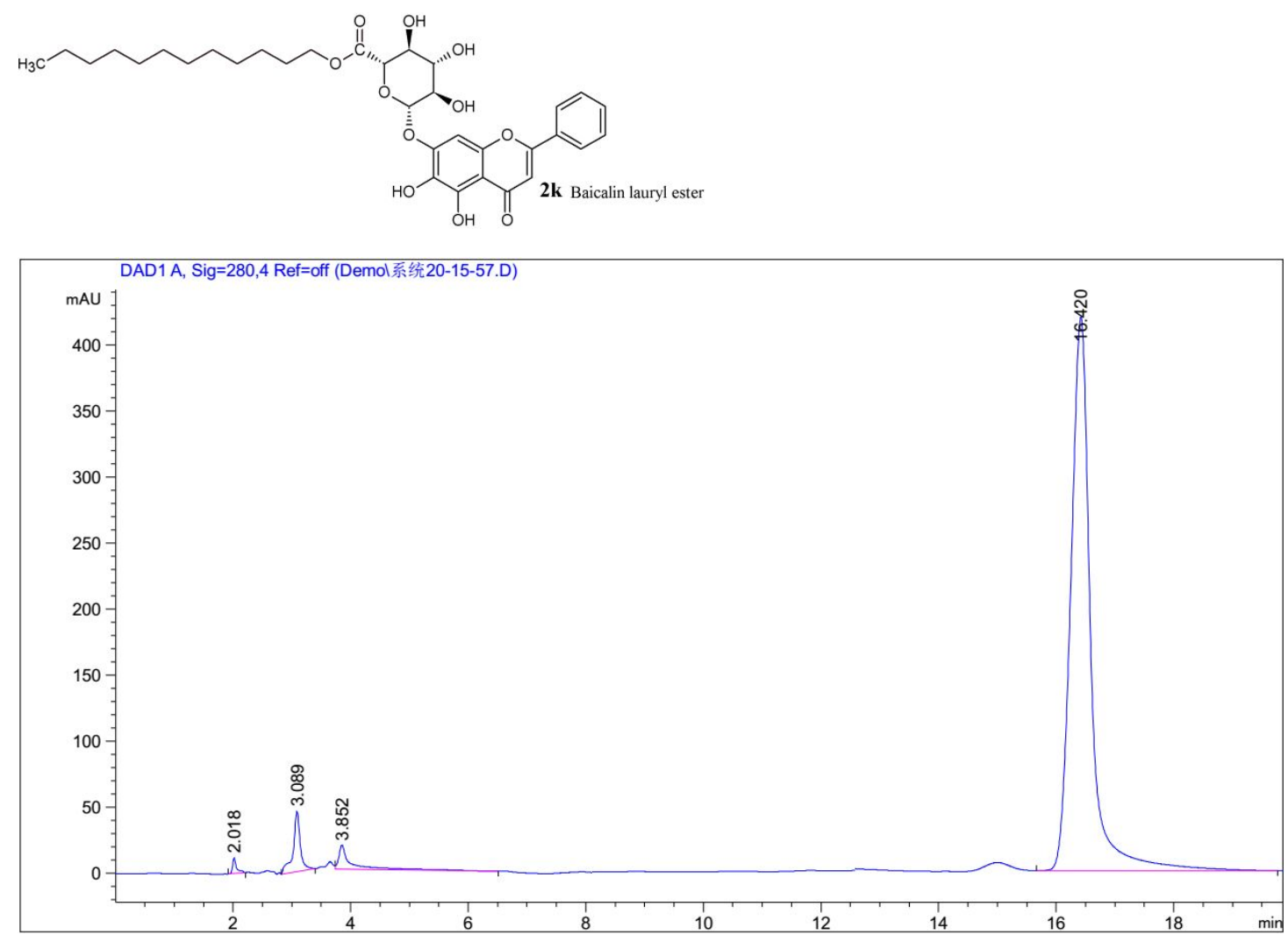

Figure S37. HPLC chromatography result of compound 2k

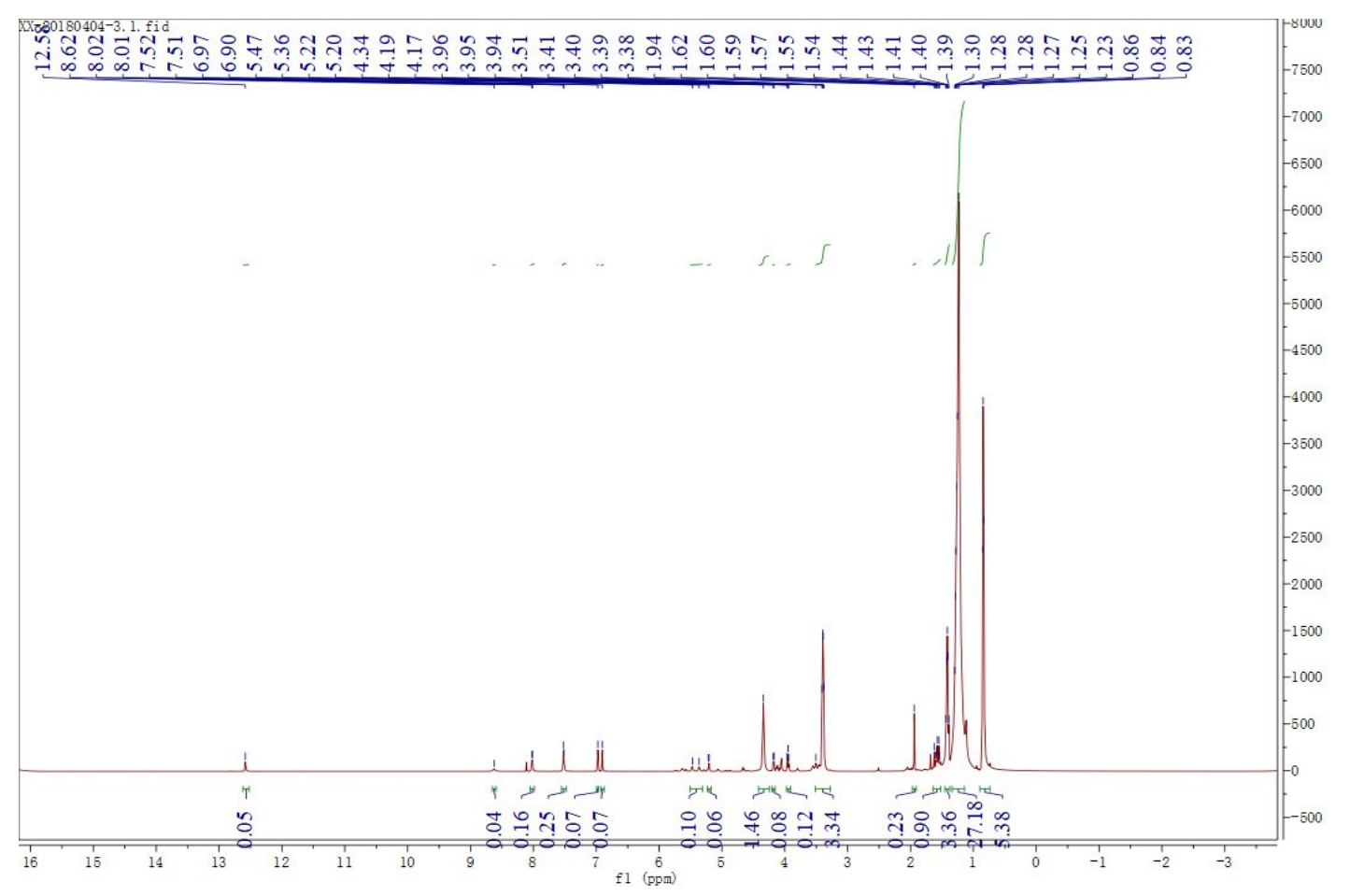

Figure S38. ${ }^{1} \mathrm{H}$ NMR spectrum for compound $2 \mathbf{k}$ in DMSO- $d_{6}(400 \mathrm{MHz})$. 


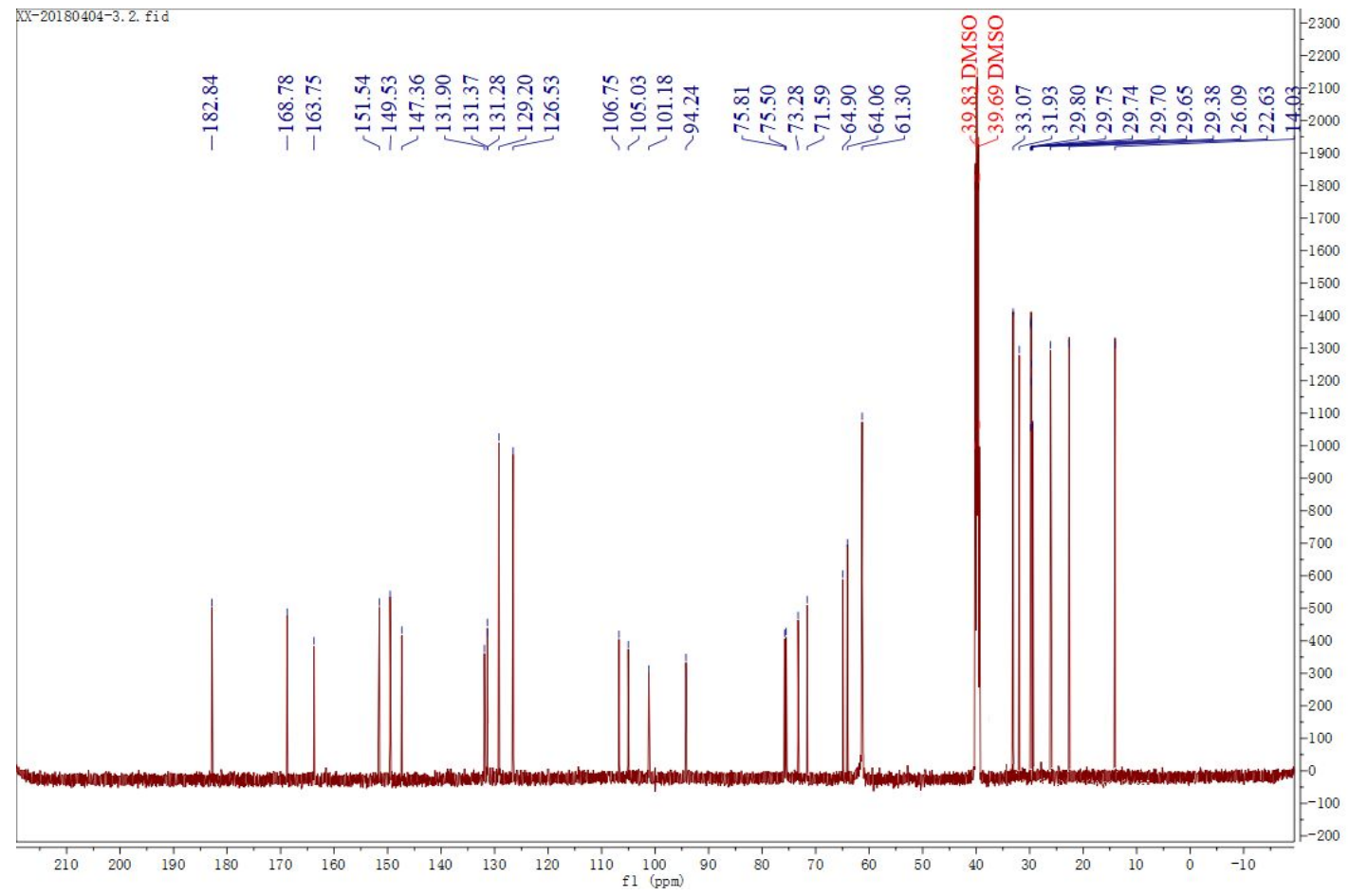

Figure S39. ${ }^{13} \mathrm{C}$ NMR spectrum for compound $\mathbf{2 k}$ in DMSO- $d_{6}(100 \mathrm{MHz})$. 